\title{
The genomic basis of the plant island syndrome in Darwin's giant daisies
}

\section{[Longer title]A chromosome-resolved polyploid assembly for Darwin's giant daisy radiation} (Scalesia, Galápagos) reveals the genomic basis of the plant island syndrome

José Cerca*,1, Bent Petersen ${ }^{2,3}$, José Miguel Lazaro Guevara ${ }^{4}$, Angel Rivera-Colón ${ }^{5}$, Siri Birkeland6, 7, Joel Vizueta8, Siyu Li ${ }^{9}$, João Loureiro10, Chatchai Kosawang11, Patricia Jaramillo Díaz ${ }^{12,13}$, Gonzalo Rivas-Torres ${ }^{14,15,16}$, Mario Fernández-Mazuecos ${ }^{17}$, Pablo Vargas ${ }^{18}$, Ross McCauley ${ }^{19}$, Gitte Petersen ${ }^{20}$, Luisa Santos-Bay², Nathan Wales ${ }^{21}$, Julian Catchen ${ }^{5}$, Daniel Machado ${ }^{22}$, Michael D. Nowak ${ }^{7}$, Alexander Suh ${ }^{23,24}$, Neelima Sinha ${ }^{9}$, Lene R. Nielsen ${ }^{11}$, Ole Seberg25, M. Thomas P. Gilbert $^{1,2}$, James H. Leebens-Mack ${ }^{26}$, Loren Rieseberg ${ }^{4}$, Michael D. Martin*,1

1 - Department of Natural History, NTNU University Museum, Norwegian University of Science and Technology, Trondheim, Norway 2 - Centre for Evolutionary Hologenomics, The GLOBE Institute, Faculty of Health and Medical Sciences, University of Copenhagen, Øster Farimagsgade 5, 1353 Copenhagen, Denmark 3 - Centre of Excellence for Omics-Driven Computational Biodiscovery, Faculty of Applied Sciences, AIMST University, Kedah, Malaysia

4 - Department of Botany and Biodiversity Research Centre, University of British Columbia, Vancouver, BC V6T 1Z4, Canada

5 - Department of Evolution, Ecology, and Behavior, University of Illinois at Urbana-Champaign, IL, USA

6 - Department of Chemistry, Biotechnology and Food Science, Norwegian University of Life Sciences, Ås, Norway

7 - Natural History Museum, University of Oslo, Oslo, Norway

8 - Villum Centre for Biodiversity Genomics, Section for Ecology and Evolution, Department of Biology, University of Copenhagen, Universitetsparken 15, 2100 Copenhagen, Denmark 9 - Department of Plant Biology, University of California, Davis, Davis, CA 95616 10 - Centre for Functional Ecology, Department of Life Sciences, University of Coimbra, Calçada Martim de Freitas 3000-095 Coimbra, Portugal

11 - Department of Geosciences and Natural Resource Management, University of Copenhagen, Rolighedsvej 23, 1958, Frederiksberg C, Denmark

12 - Estación Científica Charles Darwin, Fundación Charles Darwin, Santa Cruz, Galápagos, Ecuador

13 - Department of Botany and Plant Physiology, University of Malaga, Spain.

14 - Colegio de Ciencias Biológicas y Ambientales COCIBA \& Galapagos Institute for the Arts and Sciences GAIAS, Universidad San Francisco de Quito USFQ, Quito, Ecuador 15 - Estación de Biodiversidad Tiputini, Colegio de Ciencias Biológicas y Ambientales, Universidad San Francisco de Quito USFQ, Quito, Ecuador 16 - Estación de Biodiversidad Tiputini, Colegio de Ciencias Biológicas y Ambientales, Universidad San Francisco de Quito - USFQ, Quito, Ecuador 17 - Departamento de Biología, Universidad Autónoma de Madrid, 28049 Madrid, Spain 18 - Departamento de Biodiversidad y Conservación, Real Jardín Botánico (RJB-CSIC), Plaza de Murillo 2, 28014 Madrid, Spain 19 - Department of Biology, Fort Lewis College, Durango, C0 81301, USA 20 - Department of Ecology, Environment and Plant Sciences, Stockholm University, SE-106 91 Stockholm, Sweden 
48

49

50

51

52

53

54

55

56

57

58

59

60

61

62

63

64

65

66

67

68

69

70

71

72

73

74

75

76

77

78

79

80

81

82

83

84

85

86

87

88

89

21 - Department of Archaeology, University of York, York, UK

22 -Department of Biotechnology and Food Science, Norwegian University of Science and

Technology, Trondheim, 7491, Norway.

23 - School of Biological Sciences, University of East Anglia, Norwich Research Park, NR4 7TU,

Norwich, UK

24 - Department of Organismal Biology, Evolutionary Biology Centre (EBC), Science for Life

Laboratory, Uppsala University, 75236 Uppsala, Sweden

25 - The Natural History Museum of Denmark, University of Copenhagen, Denmark

26 - Department of Plant Biology, University of Georgia, Athens GA 30602, U.S.A.

* Corresponding authors: José Cerca jose.cerca@gmail.com; Michael D. Martin

mike.martin@ntnu.no

\section{Abstract}

Oceanic archipelagos comprise multiple disparate environments over small geographic areas and are isolated from other biotas. These conditions have led to some of the most spectacular adaptive radiations, which have been key to our understanding of evolution, and offer a unique chance to characterise the genomic basis underlying rapid and pronounced phenotypic changes. Repeated patterns of evolutionary change in plants on oceanic archipelagos, i.e. the plant island syndrome, include changes in leaf morphology, acquisition of perennial life-style, and change of ploidy. Here, we describe the genome of the critically endangered and Galápagos endemic Scalesia atractyloides Arnot, obtaining a chromosomeresolved 3.2-Gbp assembly with 43,093 candidate gene models. Using a combination of fossil transposable elements, $k$-mer spectra analyses and orthologue assignment, we identify the two ancestral subgenomes and date their divergence and the polyploidization event, concluding that the ancestor of all Scalesia species on the Galápagos was an allotetraploid. There are a comparable number of genes and transposable elements across the two subgenomes, and while their synteny has been mostly conserved, we find multiple inversions that may have facilitated adaptation. We identify clear signatures of selection across genes associated with vascular development, life-growth, adaptation to salinity and changes in flowering time, thus finding compelling evidence for a genomic basis of island syndrome in Darwin's giant daisy radiation. This work advances understanding of factors influencing subgenome divergence in polyploid genomes, and characterizes the quick and pronounced genomic changes in a specular and diverse radiation of an iconic island plant radiation.

\section{Introduction}

As naturalists set sail to explore the world, the distinctiveness of insular species stood out from the remaining biota. The collections carried out in the Galápagos, Cape Verde and Malay archipelagos were key for the development of the theory of natural selection (C. Darwin 1859; B. Y. C. Darwin et al. n.d.) and biogeography (Wallace 1962). More recently, Ernst Mayr's work, which set the scene for the modern synthesis (Mayr 1942), focused heavily on island biota (Emerson 2008). The central role of remote archipelagos in our understanding of evolution is not coincidental. Organisms colonizing these regions encounter highly distinct microenvironments that provide abundant ecological niches and thus ideal conditions for rapid adaptive radiation (Lomolino, Riddle, and Whittaker 2017). The 'island syndrome hypothesis' 
90 predicts the repeated and pronounced phenotypic shifts that species may undergo after

91 colonizing islands, as a result of novel selective pressures and empty ecological space (Baeckens

92 and Van Damme 2020). While the island syndrome hypothesis has been well established (Burns

93 2019; Baeckens and Van Damme 2020), its integration with genomic evidence still lags. For

94 instance, while body size differences in animal lineages are the textbook example of an island

95 syndrome (e.g., pygmy mammoths and giant tortoises), the extent to which these changes are

96 hereditary (genetic) or induced by different food sources (diet) has yet to be documented for

97 many lineages. Considering the rapid and drastic changes characteristic of these radiations, it

98 can be expected that rearrangements in genome structure contribute to the adaptation to novel

99 environmental conditions.

Because the most prominent examples of adaptive radiation and island syndromes feature animal lineages, such as Darwin's finches, our understanding of these phenomena in plant lineages lags (Burns 2019). As plants colonize archipelagos, they typically and repeatedly undergo shifts in leaf morphology, dispersal ability, lifespan and size (Burns 2019). This is exemplified by the daisy family (Asteraceae) which has radiated in Hawai'i (Bidens radiation and silversword radiations) (Knope et al. 2012; Baldwin and Sanderson 1998; Knope et al. 2020), Macaronesia (Sonchus, Tolpis, Argyranthemum and Cheirolophus radiations) (S. C. Kim et al. 1996; Gruenstaeudl, Santos-Guerra, and Jansen 2013; Vitales et al. 2014; White et al. 2018, 2020), Juan Fernández (Erigeron and Robinsonia radiation), Ryukyu (Ainsliaea radiation) (Mitsui and Setoguchi 2012), Tristan da Cunha (Commidendrum and Melanodendron radiation) (Eastwood, Gibby, and Cronk 2004), Mauritius (Psiadia radiation) (Besse et al. 2003), and Polynesia (Tetramolopium radiation)(Whitkus 1998).

One iconic, yet understudied, plant radiation is the remarkable diversification of daisies in the genus Scalesia (Blaschke and Sanders 2009; Fernández-Mazuecos et al. 2020; Crawford et al. 2009; U. Eliasson and U 1974). This genus consists of ca. 15 species, which have colonized moist forest, littoral, arid, dry forest, volcanic soil, lava gravel and fissured environments across varied elevations (Itow 1995; Blaschke and Sanders 2009). This adaptive ability has been linked to Scalesia's exceptional variation in leaf morphology, which may be associated with the adaptation to dry and humid environments (Stöcklin 2009; Fernández-Mazuecos et al. 2020), capilum morphology and habit. The outstanding phenotypic and ecological variation has led previous authors to refer to it as the 'Darwin finches of the plant world' (Stöcklin 2009). All Scalesia species are ancestrally tetraploid $(2 n=4 x=68$ ) (Ono 1967; Uno Eliasson 1974), and the polyploid genetics may have provided the genetic grist for adaptive radiation, as has been speculated for other island floras (Meudt et al. 2021).

Here, we describe a high-quality chromosomal reference genome assembly and annotation for Scalesia atractyloides. This species was chosen since it is a critically endangered species and because it belongs to the most basal lineage in the endemic radiation (FernándezMazuecos et al. 2020). A chromosome-resolved assembly has allowed us to identify and separate the two ancestral genomes that united in the polyploidization event, and to compare gene and transposable element distribution across and between these subgenomes. Annotation of genes using PacBio IsoSeq RNA afforded a high-quality annotation of the genome, and the detection of selection and gene-family expansions that implicate the genomic basis for island syndrome traits in plants. 


\section{Results and discussion}

134 Genome assembly, annotation, and quality control

A

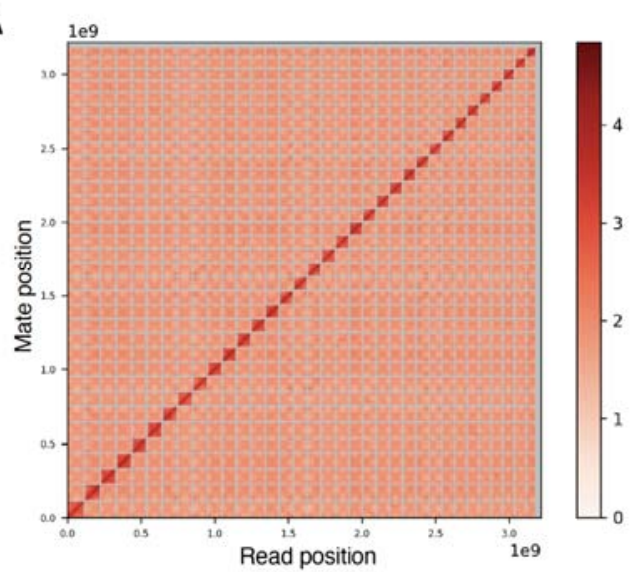

B

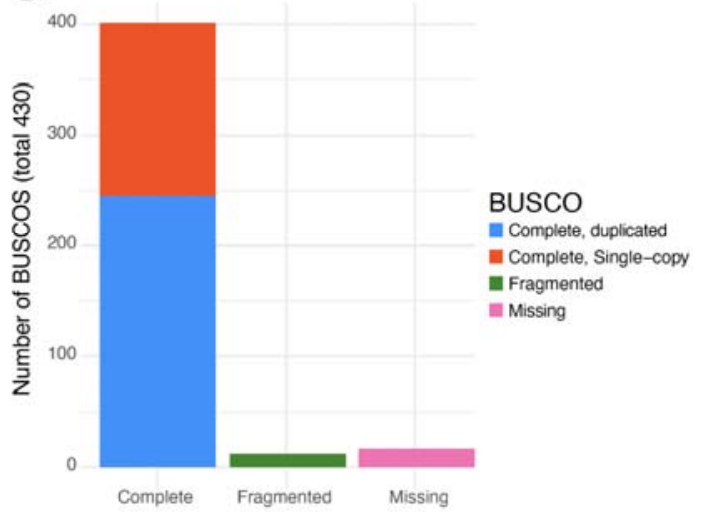

Figure 1. Chromosome-resolved assembly of the Scalesia atractyloides nuclear genome. A) Link density histogram, with 34 linkage groups (chromosome models) identified by contiguity ligation sequencing. The $\mathrm{x}$ and $\mathrm{y}$ axes show mapping positions of the first and second read in read pairs. B) Viridiplantae BUSCO set, which offers a characterization of universally conserved orthologue genes.

The Scalesia atractyloides genome assembly is of remarkably high contiguity (Figure 1A), consisting of 3,216,878,694 base pairs ( $3.22 \mathrm{Gbp}$ ) distributed over 34 chromosome models, in line with previous cytological evidence (Spring, Heil, and Vogler 1997; Uno Eliasson and Others 1974; Schilling, Panero, and Eliasson 1994). The $\mathrm{N}_{90}$ was of 31 , corresponding to all but the three smallest chromosomes $(\mathrm{n}=34)$ and $\mathrm{LN}_{90}$ was $81.66 \mathrm{Mbp}$. Flow cytometry estimates (Supplementary Information; Supplementary Table 01), however, suggest a genome size of ca. $3.9 \mathrm{Gbp}$, and thus $\sim 700 \mathrm{Mbp}$ were likely collapsed by the assembler or removed by purgehaplotigs (Peona, Weissensteiner, and Suh 2018). Despite this likely collapse of repeats, we were able to annotate $76.22 \%$ of the genome as repeats, which were masked by RepeatMasker ( $2.5 \mathrm{Gbp}$ ). Considering the whole genome, $47.9 \%$ of the genome was composed of long terminal repeat (LTR) retroelements, of which $16.2 \%$ were Copia and $31.54 \%$ Gypsy elements (Supplementary Information; Supplementary Table 2), and 26.32\% were unclassified repeats.

The IsoSeq transcriptome recovered 46,375 genes and 224,234 isoforms (Supplementary Information; Supplementary Figure 01). Using this as evidence and ab initio models, we retrieved 43,093 genes from the annotation. Of the 430 Viridiplantae odb 10 BUSCO groups used in a search of the genome (Figure 1B), 401 were found as complete (93.3\%), of which 245 were found as duplicate (57\%), 156 as complete and single-copy (36.3\%), and 12 as fragmented (2.8\%). Only 17 were absent (3.9\%). When running OrthoFinder including Scalesia and five other Asteraceae chromosome-resolved assemblies, we found that $34 \%$ of all the orthogroups included genes from the five genomes. This overlap indicates a high-quality gene annotation (Supplementary Information; Supplementary Figure 02). The proportion of annotated repeats and number of genes is within the variation reported for Asteraceae. For 
bioRxiv preprint doi: https//doi.org/10.1101/2022.0126.477903. this version posted January 28,2022 . The copyright holder for this preprint (which was not certified by peer review) is the author/funder, who has granted bioRxiv a license to display the preprint in perpetuity. It is made available under aCC-BY-NC 4.0 International license.

166 instance, the assembly of the closely related sunflower (Helianthus annuus) reference genome
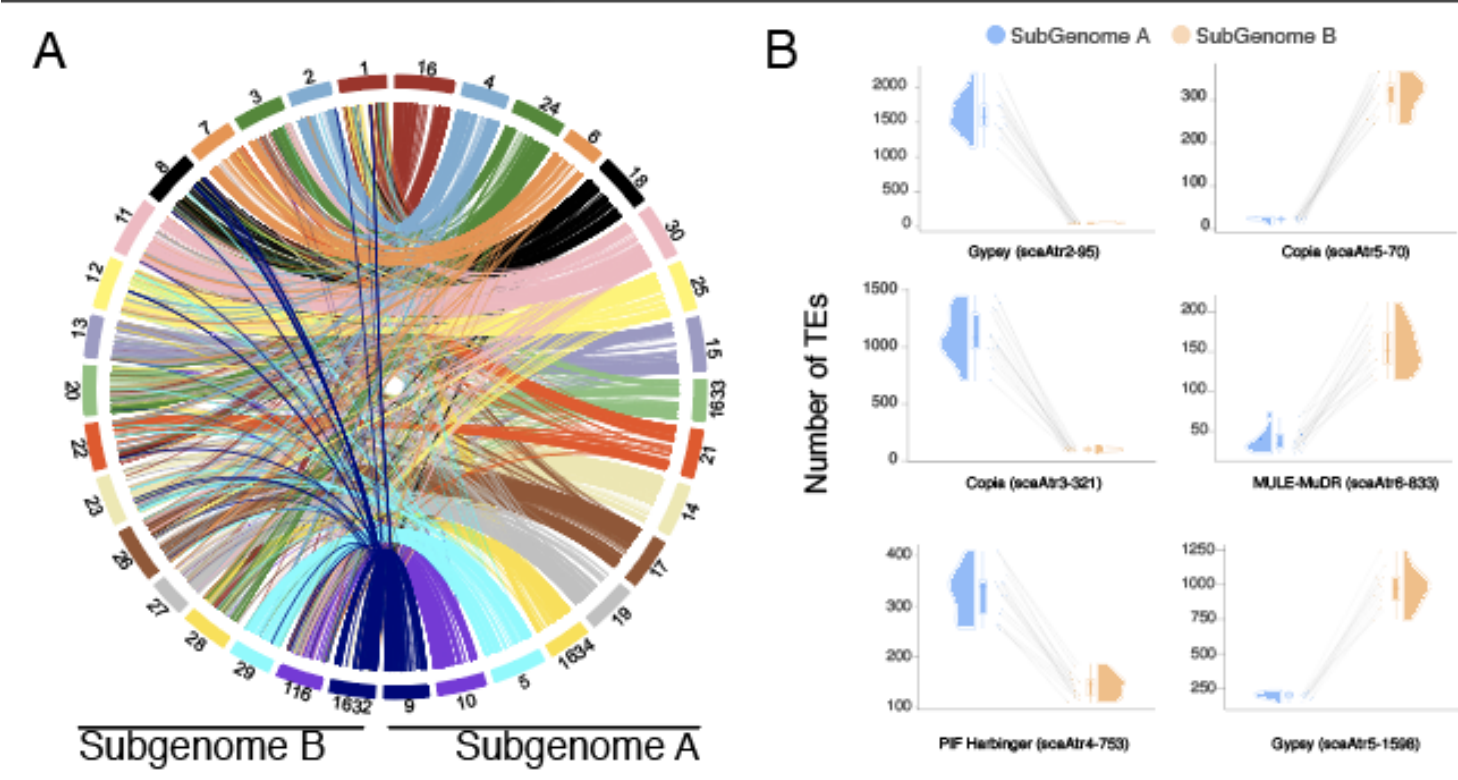

C

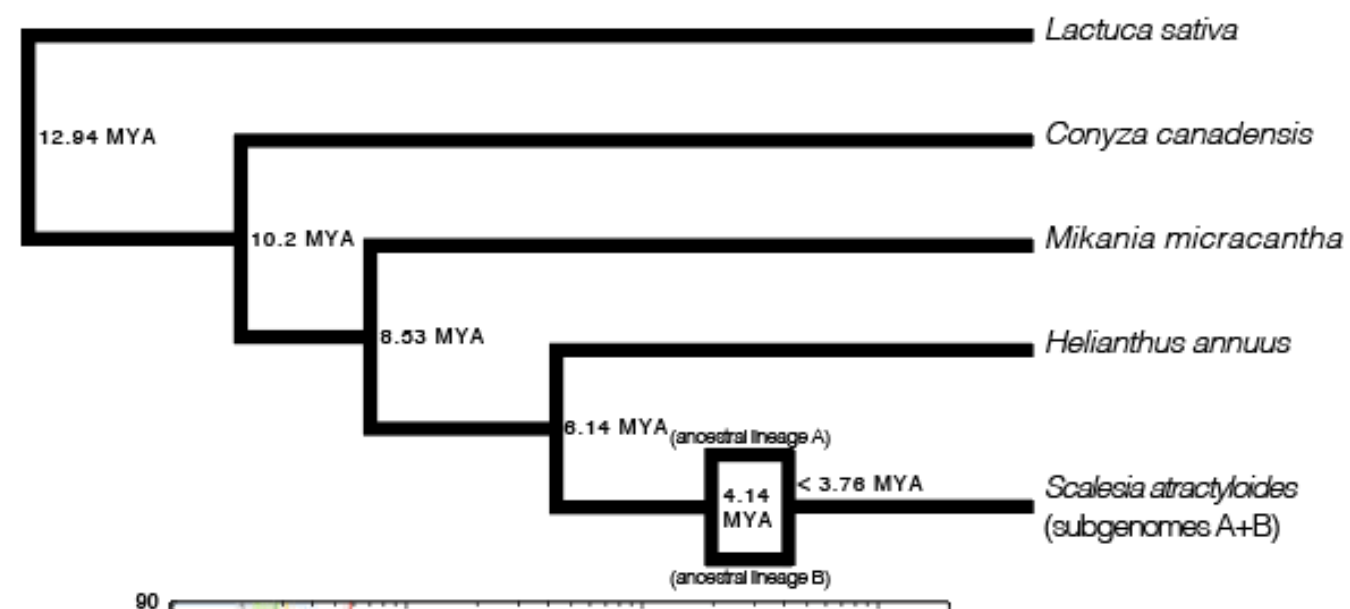

D

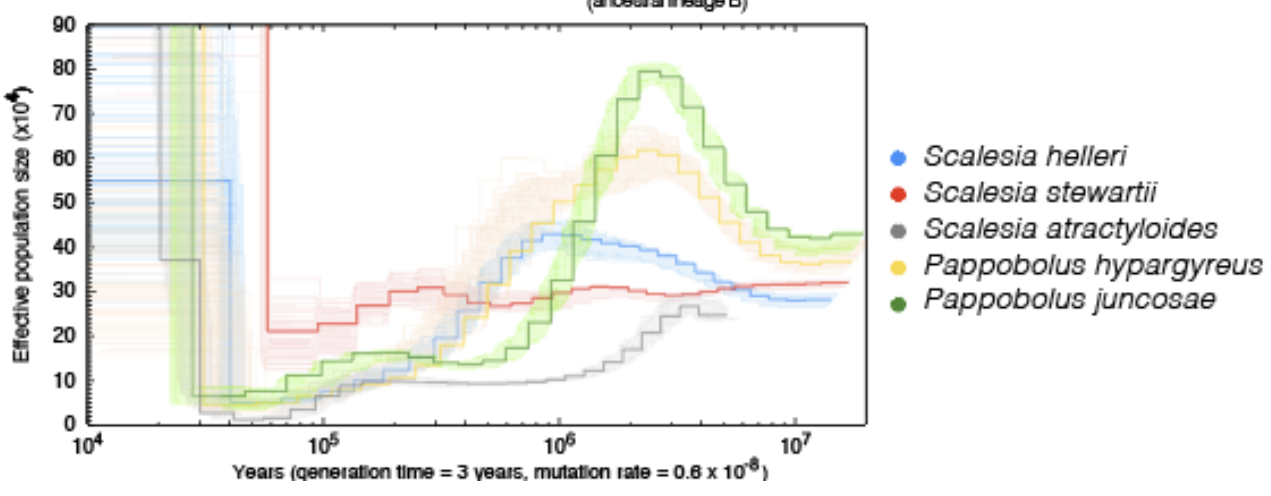


174 Figure 2. Subgenomes and evolutionary history of Scalesia. A) Circos plots displaying the 34

175 pseudo-chromosomes in the assembly. Pairs are organized to the left and right from the top, and

176 have the same colour-coding; B) Families of transposable elements (TEs) that are differently

177 represented on each subgenome. These TE families were likely active while the two

178 subgenomes were separated and thus confirm subgenome identification. Each data point

179 corresponds to a chromosome in a subgenome (subgenome A in blue and B in orange).

180

181

182

183

184

185 Asteraceae genome assemblies. Node ages are provided to the right of each node, as well as the predicted time for the polyploidization event. D) Pairwise sequentially Markovian coalescent (PSMC) estimation of the demographic history of Scalesia atractyloides, two other Scalesia species, and two members of the Pappobolus genus, which is the sister taxon to Scalesia.

The identification of subgenomes was carried in two steps. On the first step, we assigned the 34 chromosomes into 17 homeolog pairs by identifying and mapping duplicated conserved orthologous sequences (COS; Supplementary Information; Supplementary Table 03; Figure 2 A). This step only allowed the identification of pairs (homeologs), and did not allow the assignment of subgenome identity within pairs. Homeolog exchanges were therefore not a concern here (Edger et al. 2018). On the second step, we used the $k$-mer spectrum to identify 'fossil transposable elements', which are transposable elements that were replicating while both subgenomes were separate (i.e. after the speciation event, before the polyploidization event). Since different genomes accumulate different transposable elements, we hypothesized that some transposable element families in either subgenome will maintain frequency biases (Session et al. 2016; Mitros et al. 2020). In short, transposable element families active before the divergence of the two parental lineages are predicted to be approximately equally represented in either subgenome, whereas elements activated after the divergence of the parental species are predicted to be differently represented on the Scalesia subgenomes. Using the $k$-mer spectrum, we selected k-mers that were in high numbers (i.e. repeats/TEs) and unevenly represented between chromosome-pairs identified in the previous step (i.e. active during the separation period). Using this selection of $k$-mers we ran a hierarchical clustering approach that grouped chromosomes into two groups (two subgenomes; Supplementary Information; Supplementary Figure 03). To confirm this assignment, we explored the output from RepeatMasker, finding transposable element families unevenly represented across subgenomes (Figure 2B), as predicted. The identification of differently represented transposable element families also provides compelling evidence that the Scalesia radiation is of allopolyploid lineage. Island floras are characterized by a high degree of paleo-allopolyploids, where the variation brought forth by ploidy may underpin the diversification to multiple environments (Julca et al. 2020; te Beest et al. 2012) - a scenario which is line with the evolutionary history of Scalesia.

Using four other chromosome-level assemblies from Asteraceae (Helianthus annuus, Conyza canadensis, Mikania micrantha, and Lactuca sativa) and the two subgenomes, we estimated groups of orthologous genes using OrthoFinder. We obtained 710 orthogroups in which each genome had only a single member, tolerating no missing data, and used this data to construct a phylogenetic tree. The tree topology agrees with the placement of the Asteraceae lineages from a recent and comprehensive set of genomic analyses (Mandel et al. 2019). Dating of this tree was done by constraining the node separating the Scalesia subgenomes and Helianthus as 6.14 Mya (Figure 2C), following recent literature (Mandel et al. 2019). This suggests that the subgenomes diverged from their MRCA roughly 4.14 Mya, but the separation of the ancestral lineages only lasted $\sim 0.5 \mathrm{My}$, as calculated by LTR-family divergence 
(Supplementary Information; Supplementary Figure 04). Specifically, the ancestral genomes reunited in a single polyploid genome at least 3.76 Mya (Figure 2C; Supplementary Information; Supplementary Figure 04). These dates are concordant with the PSMC analysis which roughly indicate that the three Scalesia species had concordant population sizes of 250,000-300,000 circa 4 MYA (Figure 2D). Mismatches between the three genomes could result from variation in generation time in Scalesia (see results and discussion below), and bottlenecks suffered by populations as a result of climatic shifts in the Galapagos (Whittaker, School of Geography Robert J Whittaker, and Fernandez-Palacios 2007). These estimates are concordant with a recent dating analysis that estimated the divergence between Pappobolus and Scalesia occurred 3 Mya (Fernández-Mazuecos et al. 2020).
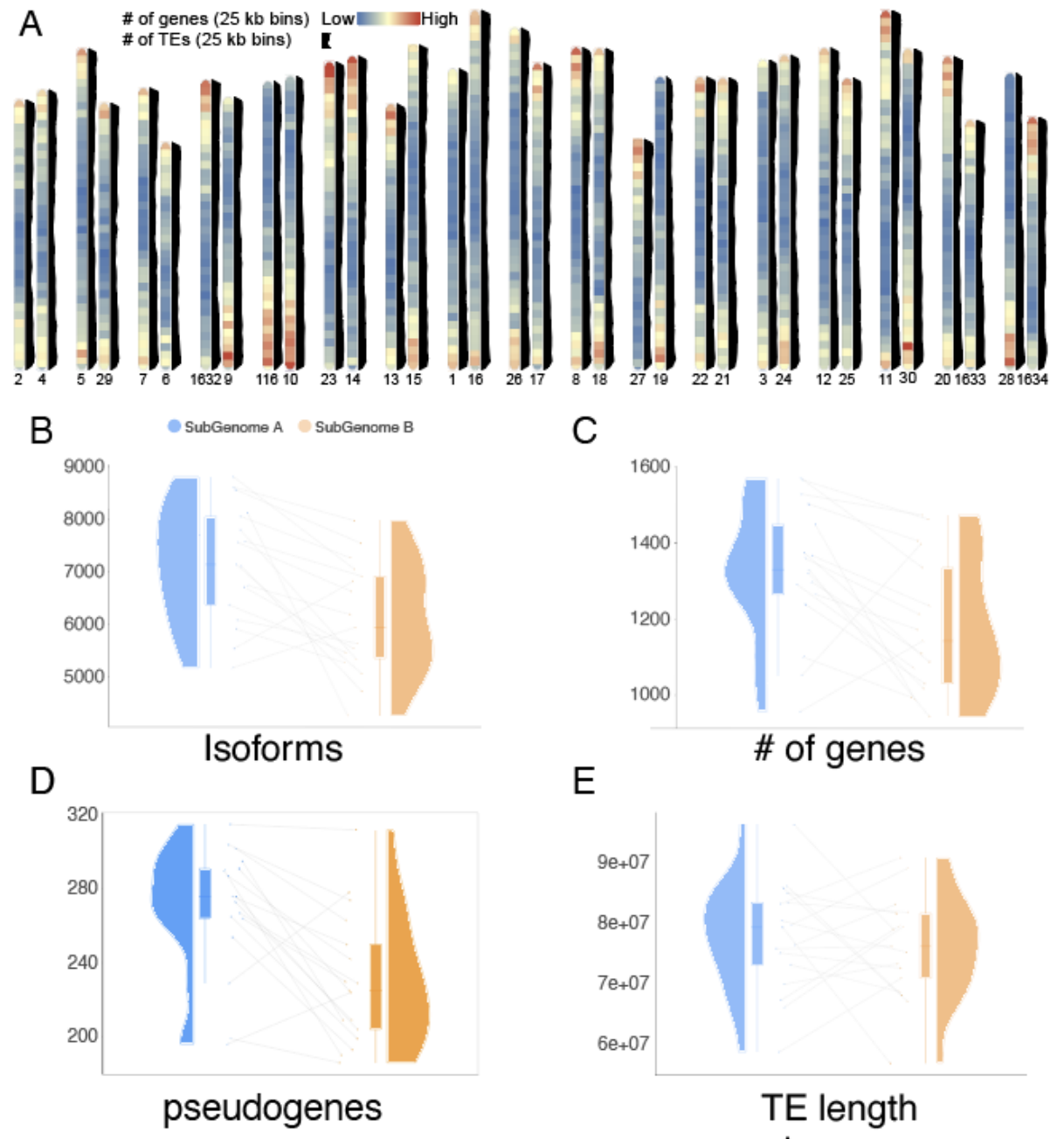

C

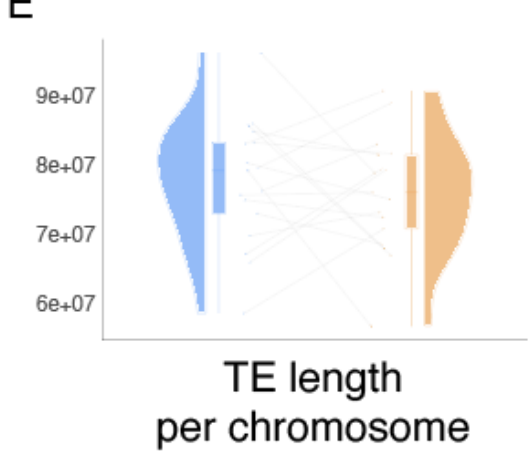

Figure 3. Subgenome evolution and characterization. A) Ideogram with gene and transposable element distribution in 25 -kbp bins. Gene density is plotted in chromosome representations and transposable element distribution is plotted to the side of each chromosome in black. Chromosomes are arranged in homoelogous pairs. B) Number of isoforms detected for each subgenome. Each data point corresponds to a chromosome in a subgenome (subgenome A in blue and B in orange). Chromosome pairs are linked by grey lines; C) Number 
240 of genes detected for each subgenome; D) Number of pseudogenes detected for each

241 subgenome; E) Length of transposable elements detected for each subgenome.

242

243

244

245

246

247

248

249

250

251

252

253

254

255

256

257

258

259

260

261

262

263

264

265

266

267

268

269

270

271

272

273

274
The identification of subgenomes allowed comparing the genes and transposable element distribution across chromosome pairs. We find that gene density is highest near the telomeres on both subgenomes, while transposable elements are more evenly distributed throughout chromosomes (Figure 3A). This even distribution of transposable elements is different from most other vascular plants, in which transposable element load is highest near the center and decreases towards the ends of the chromosome, and rather is reminiscent of observations in bryophyte genomes (Diop et al. 2020; F.-W. Li et al. 2020; Lang et al. 2018). Even distributions of transposable elements were also observed in the sunflower genome (Badouin et al. 2017), which may be indicative of particular transposable element regulation in the Heliantheae.

As two genomes unite to form a single hybrid genome, an accommodation of the two subgenomes, the process of 'diploidization' takes place (Bird et al. 2018; Freeling, Scanlon, and Fowler 2015; Wolfe 2001). This process can occur very quickly, with changes in transcription between subgenomes observed in 2-3 generations (Bird et al. 2021), and result in pronounced changes in gene numbers. Whereas subgenome dominance in gene expression and retention has been documented paleopolyploid plant genomes (Alger and Edger 2020; Renny-Byfield et al. 2015; Douglas et al. 2015), Scalesia subgenomes contain roughly equal gene and isoform contents (Figure $3 \mathrm{~B}, \mathrm{C}$ ), as well as pseudogene numbers and transposable element load (Figure $3 \mathrm{D}, \mathrm{E})$. In addition to this, when running the Viridiplantae BUSCO set for each subgenome separately, we find $82.7 \%$ complete BUSCOs on subgenome A ( $76.6 \%$ single-copy, $6 \%$ duplicates), and $81.9 \%$ complete BUSCOs (77\% single-copy, $4.9 \%$ duplicates) on subgenome B. Finally, both subgenomes are roughly the same length (subgenome $A=1,629,251,263$ bp; subgenome $B=1,554,170,668 \mathrm{bp}$ ), and have retained the same number of chromosomes (Figure $3 \mathrm{~A}$ ). This indicates that during the past $\sim 3.76$ million years, during which the two subgenomes have been unified in the same organism, there has not been a drastic rearrangement of either subgenome, despite a smaller accumulation of genes and pseudogenes on subgenome A. To explain this, we speculate that Scalesia'sadaptation to insular environments has benefitted from the genetic variation and diversity stemming from the allopolyploidization event.

\section{Fast evolutionary rates in Heliantheae}




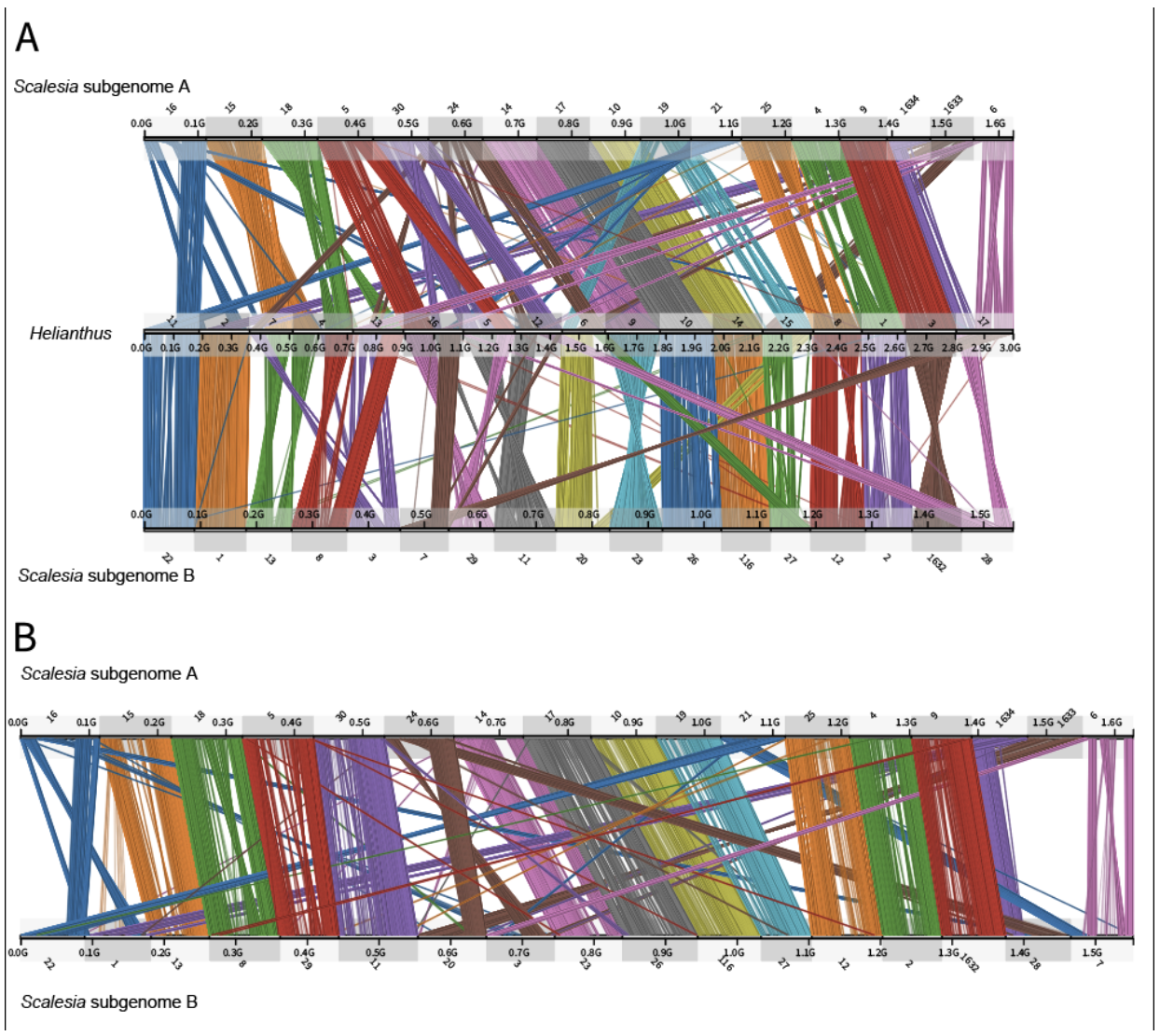

Figure 4. Chromosome stability plots reveal the role of inversions and translocations in the differential in each subgenome. A) Chromosome stability plot between the two Scalesia subgenomes and the Helianthus annuus genome. Each line connects a pair of orthologous genes, colour-coded by chromosome pair. B) Chromosome stability between the two Scalesia subgenomes. Each line connects orthologous genes in subgenome A and B, colour-coded by chromosome pair.

To further dissect the mode and tempo of polyploid subgenome evolution, we used Synolog (Catchen, Conery, and Postlethwait 2009) to create chromosome stability plots that would allow us to detect translocations and inversions and to quantify their impact (Figure 4). This method establishes clusters of conserved synteny by identifying single-copy orthologs shared between two genomes via reciprocal BLAST searching between all annotated proteincoding genes. From the identified synteny clusters, we calculated statistics on the orientation (forward/inverted) and chromosome location. We thereby classified genes into four categories: "Forward pair" (FP; i.e. not inverted, and the single-copy orthologs are in chromosomes from the same pair), "Inverted pair" (IP; i.e. inverted, and the single-copy orthologs are in chromosomes from the same pair), "Forward translocated" (FT; i.e. not inverted, and the orthologues are not in chromosomes from the same pair), "Inverted translocated" (IT; i.e. inverted, and the orthologues are not in chromosomes from the same pair). Comparing the two Scalesia subgenomes, we found 2,284 FP genes (17\%), 1,760 IP genes (13\%), 3,539 FT genes (27\%), and 5,641 IT genes (43\%), totalling 13,224 genes included in the analysis (Figure 4B). Thus, the majority of the genes have been translocated (70\%), and/or inverted (56\%). Interestingly, there is a mismatch between the length of these regions in the genome and the 
300 proportion of genes they contain. Specifically, we classified 434.6 Mbp as FP (23\%), $189 \mathrm{Mbp}$ as

$301 \mathrm{IP}(36 \%), 693,2 \mathrm{Mbp}$ as FT (31\%), and 586,5 Mbp as IT (10\%). These results are in line with the

302 inference of rapid rates of chromosomal rearrangements in the Asteraceae based on

303 comparisons of the sunflower and lettuce genomes (Badouin et al. 2017). The discordance

304 between the fraction of genes, and the fraction of genome length (e.g., IT includes $43 \%$ of the

305 genes but only occupies $10 \%$ of the genome; $46 \%$ of the subgenomes is found as inverted, but

306 contains $56 \%$ of the genes) implicates inversions as having influenced the retention of

307 duplicated genes after formation of Scalesia's allopolyploid ancestor.

308 Despite its polyploid ancestry, the Scalesiagenome has roughly the same length (3.22

$309 \mathrm{Gbp}$ ) as that of the diploid sunflower (3.6 Gbp assembly). Further, Scalesia's annotated gene

310 model number (43k) is smaller than the number of gene models annotated in the sunflower

311 genome (52k) (Badouin et al. 2017). The sunflower has 17 chromosome pairs, whereas the

312 tetraploid Scalesia has 34 chromosome pairs. This indicates that Scalesia has likely undergone a

313 reduction in genome length and gene numbers without a reduction in chromosome number.

314 This evidence is consistent with hypothesized genome miniaturization in island species (J. Suda,

315 Kyncl, and Jarolímová 2005). We cannot, however, rule out a scenario in which Helianthus has

316 increased its number of genes and genome length. 
bioRxiv preprint doi: https://doi.org/10.1101/2022.01.26.477903; this version posted January 28, 2022. The copyright holder for this preprint (which was not certified by peer review) is the author/funder, who has granted bioRxiv a license to display the preprint in perpetuity. It is made available under aCC-BY-NC 4.0 International license.
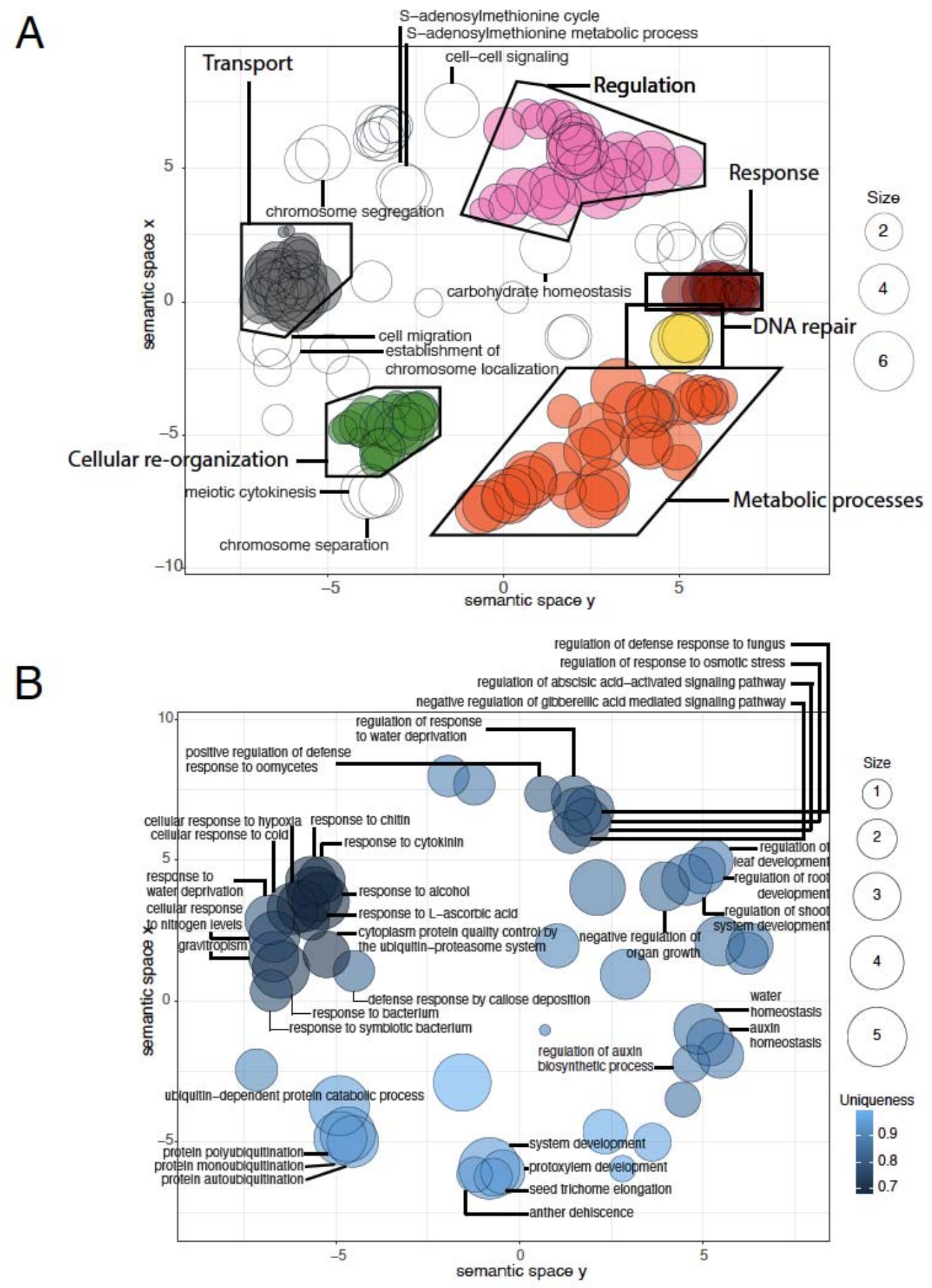

Figure 5. Positive selection and gene family expansion across the Scalesia atractyloides genome. A) GO term enrichment of the genes under selection across the genome. GO terms assigned to at least four genes are labelled. Size refers to the number of genes associated with a particular GO term. B) GO term enrichment of the genes belonging to expanded gene families across the genome according to a $C A F E$ analysis. Only GOs within a group of three or more overlapping 
326

327

328

329

330

331

332

333

334

335

336

337

338

339

340

341

342

343

344

345

346

347

348

349

350

351

352

353

354

355

356

357

358

359

360

361

362

363

364

365

366

367

368

369

370

371

372 circles are included. Uniqueness measures the degree to which a particular GO term is distinct relative to the whole list.

We identified 920 genes under selection $(p<0.05)$ in the Scalesia genome, after correcting $\mathrm{dN} / \mathrm{dS}$ ratios using a Holm-Bonferroni FDR correction. To understand their function, we extracted the functional annotation using a Gene Ontology (GO) term enrichment analysis, and the results were visualised using Revigo. GO classifications relating to many metabolic processes under selection (Figure 5A, orange group), cellular reorganization (green group), DNA repair (yellow group), response to protein folding (maroon group), and regulation (regulation of metabolic processes, translation, gene expression, translation, nuclear division, chromosome segregation, among others; pink group; Figure 5A; Supplementary Information; Supplementary Tables 04-6). Genes inferred to have evolved under positive selection are also associated with meiosis, chromosome arrangement, and chromatin status (meiotic cytokinesis, establishment of chromosome location, chromosome separation, and chromosome segregation, among other GO classifications; Figure 5A, Supplementary Information; Supplementary Tables 04-6), and this may indicate selection at genes associated with the coexistence of two genomes.

To chart the landscape of Scalesia's adaptive revolution, we randomly selected 100 genes under selection and performed a systematic investigation of homolog function in Arabidopsis thaliana literature. The classification of these genes into non-mutually exclusive categories showed 23 genes implicated in photosynthesis and leaf morphology/metabolism, 17 in stress response, 15 in fertility and reproductive organ function, 13 in life-phase transition and growth, 11 in the embryo, 10 in root functions, eight in immunity, seven in cellular functions (mitochondrial functions, cytoplasm, DNA repair, nucleus, cell elongation or autophagy), five in meiosis/mitosis, three in the vascular system, two in general metabolism, and one in methylation (Supplementary Information; Supplementary Table 07). As leaf morphology was an important innovation in the Scalesia radiation, it is particularly interesting that we found selection on potential regulators of leaf morphology, including genes well known to determine leaf cell number in $A$. thaliana (E2F1) (Őszi et al. 2020; Berckmans et al. 2011), cell fate in leaves (YABBY5) (Kojima et al. 2011; Husbands et al. 2016), leaf senescence (RANBPM, LARP1C, PEN3) (Crane et al. 2019; Fu et al. 2019; Zhang et al. 2012), leaf variegation (THF1) (Ma et al. 2015; Z. Wang et al. 2016), and leaf growth (PAC) (Jörg Meurer et al. 2017; Holding 2000; J. Meurer et al. 1998). It is also interesting to note that many Scalesia genes under selection are affected by light stimulus. A STRING analysis showed that there was selection at multiple points in the light regulatory pathways including responses to R/FR and blue light responses. These include an inhibitor of red and far-red light photoreceptor (PHL) (Endo et al. 2014, 2013), a lysine-tRNA ligase that regulates photomorphogenic responses (G. Li et al. 2011), an amino acid aminotransferase-like PLP-dependent enzymes superfamily protein that is regulated under light conditions and is associated with the photorespiration process (Basset et al. 2004; Smeekens 2006), and genes for which knock-out mutants experience alterations in light reception (DJC69, COX15) (Oravecz et al. 2006; Dal Bosco et al. 2004). This may underlie the natural history observations where Scalesia individuals growing in the absence of permanent light conditions show substantially retarded growth (Lawesson 1988) or higher mortality (Rivas-Torres, unpublished data).

Many of the stress-response genes under selection in Scalesia are associated with osmotic stress in A. thaliana, concomitant with evidence that the Scalesia atractyloides habitat is characterized by arid conditions such as the Galápagos' arid zone, the littoral zone, and fissured lava areas (Blaschke and Sanders 2009; Itow 1995). For instance, we found five genes ( 
373 "Leucine-rich repeat protein kinase family protein", MPPBETA, "leaf osmotic stress elongation

374 factor 1- $\beta-1$ ", AT2G21250, VAP27-1) associated with osmotic stress (Tan et al. 2010; J. Y. Kim et

375 al. 2007; ten Hove et al. 2011; Jakoby et al. 2008; Fox et al. 2020; P. Wang et al. 2019), but also

376 heat shock proteins (Valverde, Groover, and Romero 2018), and regulators of stomatal closure

377 (THF1) (Ma et al. 2015; Z. Wang et al. 2016) under selection. Other stress-associated genes

378 under selection include those involved in response to high irradiation (ZAT10, AT1G06690,

379

380

381

382

383

384

385

386

387

388

389

390

391

392

393

394

395

396

397

398

399

400

401

402

403

404

405

406

407

408

409

410

411

412

413 DDB2) (Bittner, Hause, and Baier 2021; Kuki et al. 2020; Czarnocka et al. 2020; Kleine et al. 2007; Castells et al. 2011; Lahari et al. 2018).

Some genes under selection are associated with growth and transitions between life stages. Scalesia plants' fast rates of growth have earned them the name 'weedy trees', and these genes may regulate these plants' exceptionally fast growth and tree-like habits. We find three genes under selection that cause the transition between embryonic and vegetative traits (RING1A, SWC4, ABCI20) (A. Kim et al. 2020), and four genes that regulate flowering time in $A$. thaliana (ELF8, RING1A, Short-Vegetative-Phase, NRP1) (D. Chen et al. 2010; Shen et al. 2014; J. Li et al. 2017; An et al. 2020; Gómez-Zambrano et al. 2018), and height or size of the plant (CLAVATA, GH9C2, ELF8, NSL1, TUA6) (Glass et al. 2015; Markakis et al. 2012; Noutoshi et al. 2006; Fukunaga et al. 2017; Singh et al. 2021; Fal et al. 2017; He 2004; Hoson et al. 2014; Xiong et al. 2013; Whitewoods et al. 2020).

Genes under selection include some genes associated with increased sensitivity to bacteria and fungi (JAM3, ABCG16, NMT1, AT5G05790) (Kapos et al. 2015; Kessler et al. 2010; Swain, Singh, and Nandi 2015; Sasaki-Sekimoto et al. 2013; Ji et al. 2014) as well as WRKY70, which is central to immunity in A. thaliana (Noh et al. 2021; H. Liu et al. 2021; S. Chen et al. 2021). These may indicate the importance of rapid evolutionary response to the new enemies and symbionts colonizing plants encounter upon their arrival to volcanic archipelagos.

Finally, we assessed the expansion and contraction of gene families in the Scalesia genome, finding a total of 37 significantly contracted families and 26 significantly expanded families (Figure 5B). GO enrichment testing of the expanded families uncovered significantly enriched functions associated with vascularization (secondary cell wall biogenesis, shoot system development, negative regulation of organ growth, xylem vessel member cell differentiation, protoxylem development), likely associated with plant growth in Scalesia (Fernández-Mazuecos et al. 2020). We also find evidence of evolutionary responses to aridity and changes in osmotic pressure in significantly expanded families (regulation of stomatal closure, response to water deprivation, response to osmotic stress, water homeostasis), similar to the genes under selection (Figure 5B). Interestingly, we detect contraction in gene families with GO terms associated with tree habits (shoot system development, regulation of organ growth, regulation of root development, xylem vessel member cell differentiation, gravitropism), adaptation to arid environments (water deprivation, stomatal closure, regulation to osmotic stress) and cold tolerance (cellular response to cold; Supplementary Information; Supplementary Tables 08-13). While this may seem contradictory, it suggests that different families have redundant functions, and the expansion of a family may lead to redundancy in another family and consequent gene loss through pseudo-gene formation.

\section{Conclusions}

In this study, we were able to elucidate patterns of genome evolution in the critically

416 endangered Darwin's giant daisy tree (Scalesia atractyloides) by attaining a chromosome- 
417 resolved genome and by subsequently identifying two ancient genomes underlying its polyploid

418 state. We found that both subgenomes retain a relatively similar number of genes as well as

419 other genetic features, such as pseudogenes and transposable elements, which lead us to

420 speculate on the role of insular evolution underlying these changes. Moreover, we uncovered

421 the role of inversions in gene accumulation, suggesting these have played an important role in

422 the maintenance of genes in subgenomes, and found a relatively unique pattern of transposable

423 element accumulation within flowering of plants. Ultimately, expanded gene families and genes

424 under positive selection indicate the first and solid evidence for genomic island syndrome in a

425 plant, revealing an underlying genomic basis of the outstanding phenotypic variation in Scalesia.

426 Methods

427 Plant material, flow cytometry, DNA extraction, library preparation and sequencing

428 Tissues used for the de-novo genome assembly and annotation were sampled from

429 living Scalesia atractyloides plant P2000-5406/C2834 cultivated in the greenhouse of the

430 University of Copenhagen Botanical Garden collections. This plant was originally germinated

431 from a seed collected from Santiago Island. Fresh tissue was collected and flash-frozen in dry ice

432 or liquid nitrogen and then stored at $-80 \mathrm{C}$ for later use.

433

434

435

436

437

438

439

440

441

442

443

444

445

446

447

448

449

450

451

To assist with sequencing coverage strategy and to inform genome assembly, we obtained estimates of genome size using flow cytometry following (Galbraith et al. 1983). Briefly, $50 \mathrm{mg}$ of freshly collected leaves from the sample material and from the reference standard (Solanum lycopersicum 'Stupické'; 2C $=1.96$ pg; (Jaroslav Dolezel, Sgorbati, and Lucretti 1992) were chopped with a razor blade in a Petri dish containing $1 \mathrm{ml}$ of Woody Plant Buffer (Loureiro et al. 2007). The nuclear suspension was filtered through a 30- $\mu$ m nylon filter, and nuclei were stained with $50 \mathrm{mg} \mathrm{ml-1}$ propidium iodide (PI) (Fluka, Buchs, Switzerland). Fifty mg ml-1 of RNase (Sigma, St Louis, MO, USA) was added to the nuclear suspension to prevent staining of double-stranded RNA. After a 5 minute incubation period, samples were analysed in a Sysmex CyFlow Space flow cytometer (532 nm green solid-state laser, operating at $30 \mathrm{~mW}$ ). At least 1,300 particles in G1 peaks were acquired using the FloMax software v2.4d (Jan Suda et al. 2007). The average coefficient of variation for the G1 peak was below $5 \%$ (mean $\mathrm{CV}$ value $=2.72 \%$ ). The holoploid genome size in mass units (2C in pg; sensu (Greilhuber et al. 2005) was obtained as follows: sample 2 C nuclear DNA content (pg) = (sample G1 peak mean / reference standard G1 peak mean) * genome size of the reference standard. Conversion into base-pair numbers was performed using the factor: $1 \mathrm{pg}=0.978 \mathrm{Gbp}$ (J. Dolezel et al. 2003). Three replicates were performed on two different days, to account for instrumental artefacts.

The commercial provider Dovetail Genomics extracted and purified high-molecularweight DNA from flash-frozen leaf tissue using the CTAB protocol, and the concentration of DNA was measured by Qubit. For long-read sequencing, they constructed a PacBio SMRT bell library ( 20kb) using the SMRTbell Template Prep Kit 1.0 (PacBio, CA, USA) following the manufacturer recommended protocol. This library was bound to polymerase using the Sequel Binding Kit 2.0 (PacBio) and loaded onto the PacBio Sequel sequencing machine using the MagBeadKit v2 (PacBio). Sequencing was performed on the PacBio Sequel SMRT cell, using Instrument Control Software v5.0.0.6235, Primary analysis software v5.0.0.6236, and SMRT Link Version 5.0.0.6792. PacBio sequencing yielded 41,322,824 reads, resulting in a total of 197fold coverage of the nuclear genome. For contiguity ligation, they prepared two Chicago libraries as described in (Putnam et al. 2016). Briefly, for each Dovetail Omni-C library, 
461 chromatic is fixed in place with formaldehyde in the nucleus and then extracted. Fixed

462

463

464

465

466

467

468

469

470

471

472

473

474

475

476

477

478

479

480

481

482

483

484

485

486

487

488

489

490

491

492

493

494

495

496

497

498

499

500

501

502

503

504

505

506

507 chromatin is digested with DNAse I, and chromatin ends are repaired and ligated to a biotinylated bridge adapter followed by proximity ligation of adapter containing ends. After proximity ligation, crosslinks are reversed and the DNA is purified. Purified DNA is then treated to remove biotin that was not internal to ligated fragments. Sequencing libraries were generated using NEBNext Ultra enzymes and Illumina-compatible adapters. Biotin-containing fragments were isolated using streptavidin beads before PCR enrichment of each library. These libraries were then sequenced on an Illumina HiSeq 2500 instrument, producing a total of $1,463,389,090$ sequencing reads.

To obtain RNA transcript sequences for annotation of the genome, we extracted RNA from five tissues (root, stem, young leaf, old leaf, and floral head) of $S$. atractyloides plant P2000-5406/C2834 using a Spectrum Plant Total RNA Kit (Sigma, USA) with on-column DNA digestion following the manufacturer's protocol. RNA extracts from all five tissues were pooled. mRNA was enriched using oligo (dT) beads, and the first strand cDNA was synthesized using the Clontech SMARTer PCR cDNA Synthesis Kit, followed by first-strand synthesis with SMART ScribeTM Reverse Transcriptase. After cDNA amplification, a portion of the product was used directly as a non-size selected SMRTbell library. In parallel, the rest of amplification was first selected using either BluePippin or SageELF, and then used to construct a size-selected SMRTbell library after size fractionation. DNA damage and ends were then repaired, followed by hairpin adaptor ligation. Finally, sequencing primers and polymerase were annealed to SMRTbell templates, and IsoSeq isoform sequencing was performed by Novogene Europe (Cambridge, UK) using a PacBio Sequel II instrument, yielding 223,051,882 HiFi reads.

\section{Genome assembly and annotation}

An overview of the bioinformatic methods is provided in IOSES GITHUBPAGE. We assembled the genome using wtdbg2 (Ruan and Li 2020), specifying a genome size of $3.7 \mathrm{Gbp}$, PacBio Sequel reads, and minimum read length of 5,000. The $w t d b g 2$ assembly consisted of contigs with $3.62 \mathrm{Gbp}$ total length. This assembly was then assessed for contamination using Blobtools v1.1.1 (Laetsch and Blaxter 2017a) against the NT database, detecting a removing a fraction of the scaffolds. This filtered assembly was used as input to and purge_dups v1.1.2, which removed duplicates based on sequence similarity and read depth (Guan et al. 2020), reducing the assembly length was reduced to $3.22 \mathrm{Gbp}$. This assembly and the Dovetail OmniC library reads were used as input data for HiRise by aligning the Chicago library sequences to the input assembly. After aligning the reads on the reference genome using bwa, HiRise produces a likelihood model for genomic distance between read pairs, and the model was used to identify misjoints, prospective joints, and make joins. After HiRise scaffolding, the $\mathrm{N}_{50}$ increased to 16, and the $\mathrm{N}_{90}$ to 31 , corresponding to all but the three smallest chromosomes $(n=34)$, while the $\mathrm{LN}_{50}$ was $94.2 \mathrm{Mbp}$ and the $\mathrm{LN}_{90}$ was $81.66 \mathrm{Mbp}$. The largest scaffold was $116.23 \mathrm{Mbp}$. In total, HiRise scaffolding joined 1,329 scaffolds (suppl, assemblathon script). We used the Assemblathon 2 script ( https://github.com/ucdavis-bioinformatics/assemblathon2-analysis ) (Bradnam et al. 2013) to assess assembly quality.

To annotate genes, we first masked repeats and low complexity DNA using RepeatMasker v4.1.1 (Smit, Hubley, and Green 2013) using the 'Asteraceae' repeat database with Repbase database. After this first round, we ran RepeatModeler v2.0.1 (Flynn et al. 2020) on the masked genome to obtain a database of novel elements (denovo). This database was subsequently used as input to RepeatMasker for a second round of masking the genome. To find gene models, we first assembled a transcriptome using PacBio HiFi data and following the 
508 IsoSeq3 pipeline (Pacific Biosciences). Processing of the RNA data involved clipping of 509 sequencing barcodes (lima v2.0.0), removal of poly(A) tails and artificial concatemers (Isoseq3

510 refine v3.4.0), clustering of isoforms (Isoseq3 cluster v3.4.0), alignment of the reads to the

511 reference genome using (pbmm2 align v1.4.0), characterization and filtering of transcripts

512 (SQANTI3 v1.0.0) (Tardaguila et al. 2018). Genome annotation was carried out using the

513 MAKER2 pipeline v2.31.9 (Holt and Yandell 2011; Moore et al. 2008), using a combination of $a b$ -

514 initio and homology-based gene predictions (using Asteraceae protein sets). Since no training

515 gene models were available for Scalesia atractyloides, we used CEGMA (Parra, Bradnam, and

516 Korf 2007) to train the ab-initio gene prediction software SNAP(Korf 2004). In addition to the

517 ab-initio features, we used the PacBio-based transcriptome as a training set for the gene

518 predictor AUGUSTUS (Keller et al. 2011), and as direct RNA evidence to MAKER2. Finally, when

519 running $M A K E R 2$ we specified, model_org=simle, softmask=1, augustus_species=arabidopsis

520 and specifying snapphmm to training of SNAP. To assess the quality of the gene models we

521 started using BUSCO and the viridiplantae odb v10 set (Simão et al. 2015; Waterhouse et al.

522 2018; Seppey, Manni, and Zdobnov 2019).

523

524

525

526

527

528

529

530

531

532

533

534

535

536

537

538

539

540

541

542

543

544

545

546

\section{Demographic reconstruction using PSMC}

To complement the $S$. atractyloides genome, we generated shotgun genomic data from DNA extracts of specimens of $S$. helleri B. L. Rob. and $S$. stewartii Riley, as well as the outgroup species Pappobolus hypargyreus and $P$. juncosae. Briefly, the $S$. helleri and $S$. stewartii specimens were extracted with a Qiagen DNeasy 96 Plant Kit. The P. hypargyreus and $P$. juncosae extracts were previously reported (Fernández-Mazuecos et al 2020). DNA extracts for these four specimens were sent to the commercial provider Novogene for dsDNA library preparation, and they were sequenced on the Illumina NovaSeq platform in 150-bp PE mode. For these sequence data, we used FastQC $\mathrm{v} 0.11 .8$ to check for quality of raw reads (Andrews 2017), identified adapters using AdapterRemovalv2.3.1, and removed them using Trimmomatic v0.39 (Schubert, Lindgreen, and Orlando 2016; Bolger, Lohse, and Usadel 2014). These sequences were then aligned to the $S$. atractyloides genome using the mem algorithm of bwa $(\mathrm{H}$. Li and Durbin 2009), and reads with a mapping quality below 30 were removed, resulting in a final high-quality sequencing depth of about $\sim 15 \mathrm{x}$. Alignments were then processed and analysed using PSMC (Heng Li and Durbin 2011). Specifically, this processing involved calling variants using the bcftools mpileup and callalgorithms, considering base and mapping qualities above 30 and read depths above 5 (Danecek et al. 2021), and posterior processing of the files using fq2psmcfa. For the PSMC run we specified a maximum of 25 iterations, initial theta ratio of 5 , bootstrap, and a pattern of " $4+25^{*} 2+4+6$ ". To plot files we used the util psmc_plot.pl specifying a generation time of 3 years and a mutation rate of $6 \mathrm{e}^{-9}$, and constrained the $\mathrm{y}$ - and $\mathrm{x}-$ axes to 50 and $20,000,000$, respectively.

\section{Determination of subgenomes, and testing for subgenome dominance}

We reasoned that homologous chromosomes would share conserved orthologue sets (COS). We used the Compositae-COS as baits (available through github.com/Smithsonian/Compositae-COS-workflow/raw/master/COS_probes_phyluce.fasta (Mandel et al. 2014), running phyluce to mine for COS in the genome assembly (Faircloth 2016; Faircloth et al. 2012). This pipeline, however, is designed for single-copy COS, and we modified the python script to provide duplicates. We then constructed a matrix of COS-assignation using double-copy COS (Supplementary Information; Supplementary Table 03). 
554 Duplicated-COS provided a solid determination of chromosome pairings but did not

555

556

557

558

559

560

561

562

563

564

565

566

567

568

569

570

571

572

573

574

575

576

577

578

579

580

581

582

583

584

585

586

587

588

589

590

591

592

593

594

595

596

597

598

599

600 reveal which member of the pair belongs to either subgenome (subgenome A or B hereafter). To distinguish this, we analysed the $k$-mer spectrum (Session et al. 2016). We hypothesized that given a period of separation between the two subgenomes, they have accumulated different repeat content and transposable elements. To do so, we ran the software Jellyfish (Marçais and Kingsford 2011) for each chromosome independently, thus obtaining a chromosome-bychromosome frequency of 13-mers. To ensure we obtained only repeats, we selected 13-mers represented only $>100$ times in each chromosome, and kept only $k$-mers which were twice represented on a member of each pair. Using $\mathrm{R}$, we computed a distance matrix and a hierarchical clustering, which neatly separated members of each pair into two groups (Supplementary Information; Supplementary Figure 03).

To confirm the quality of subgenome assignment we took two independent approaches. First, we did a circos plot using the masked regions of the genome. To do the circos plot we aligned the masked subgenomes to each other using mummer (Delcher et al. 1999; Kurtz et al. 2004), and plotted the circos using the 'Circos, round is beautiful' software (Krzywinski et al. 2009). Second, we studied transposable element representation in each subgenome benefiting from the transposable element identification done by RepeatMasker. In specific, we obtained the list of different annotated transposable elements from RepeatMasker (e.g. RTE-BovB, LINEL1, LINE-L2, Helitron, PIF-Harbinger Gypsy, Copia, CRE), and separated the families within these groups. For each family, we counted the number of elements present on each subgenome, and plotted all the families using raincloud plots (Allen et al. 2019). To visualize genes and transposable elements along chromosomes we used the R package Ideogram (Hao et al. 2020). After finding out each subgenome, we ran BUSCO separately for each subgenome as a way of understanding subgenome specific gene loss (Viridiplantae odb10 as specified above).

\section{Evolutionary history of the Scalesia atractyloides subgenomes and comparative genomics}

We searched the literature and NCBI for chromosome-level assemblies of the Asteraceae (05 / February / 2021), downloading the genomes of the sunflower (Helianthus annuus (Badouin et al. 2017)), the Canada fleabane (Conyza canadensis; (Laforest et al. 2020)), the 'mile-a-minute' weed (Mikania micrantha; (B. Liu et al. 2020)), and the lettuce (Lactuca sativa; (Reyes-Chin-Wo et al. 2017)). We downloaded the Arabidopsis thaliana genome from Arabidopsis.org.

To obtain sets of orthologous genes, we ran OrthoFinder (Emms and Kelly 2015) on predicted amino acid sequences (faa) and coding sequences (cds). Before running this software, we selected only the longest isoforms of both files, and removed sequences with stop codons. On the amino acids file we removed sequences with lengths below $30 \mathrm{bp}$ using kinfin's

filter_fastas_before_clustering.py script (Laetsch and Blaxter 2017b). We ran OrthoFinder on various combinations of the genomes, including: 1) All Asteraceae, with subgenomes separated, ( $S$. atractyloides subgenomeA, $S$. atractyloides subgenomeB, $C$. canadensis, H. annuus, L. sativa, $M$. micrantha); 2) All Asteraceae, and the Scalesia genome (S. atractyloides (complete), $C$. canadensis, $H$. annuus, L. sativa, M. micrantha); 3) A. thaliana and subgenomes ( $S$ atractyloides subgenomeA, $S$. atractyloides subgenomeB, $A$. thaliana). A representation of run 2 and its processed results using an upset plot (Conway, Lex, and Gehlenborg 2017).

To obtain a tree of the two subgenomes we ran OrthoFinder with the two subgenomes and obtained the tree of the single-copy orthologs. This tree was made ultrametric using r8s (Sanderson 2003). To date the tree, we converted branch lengths to time estimates using a calibration point of 6.14 Mya between $H$. annuus and $S$. atractyloides following recent literature 
601 (Mandel et al. 2019). To date the divergence of the subgenomes we followed the approach of 602 (Session et al. 2016; Lovell et al. 2021; Mitros et al. 2020). Briefly, this approach has a simple 603 assumption: before the speciation event (which separates the ancestral lineages) and after the 604 polyploidization event (which brings the ancestral genomes together), the accumulation of 605 transposable elements will be similar on both subgenomes. Transposable element families 606 which are equally represented on both subgenomes will therefore represent the pre-speciation and post-allopolyploidization period. We focused on long-terminal repeats (LTRs) given their prevalence along the genome. We used LTRharvest to identify LTR elements (Ellinghaus, Kurtz, and Willhoeft 2008), and LTRdisgest to process these (i.e. annotating features such as genes inside LTRs). To find these features we downloaded various PFAM domains provided in (Steinbiss et al. 2009), and downloaded "Gypsy" and "Copia" domains from the PFAM online database. We converted the domains to HMMs using hmmconvert (Eddy 1992), and added HMMs from the Gypsy Database (Llorens et al. 2011). The identification and annotation of LTRs was done for the $S$. atractyloides and $H$. annuus genomes. Instead of using the whole LTRelement (i.e. whole transposable element including repeated regions and genes insides) we used only the LTR-region (long terminal repeat), and ran OrthoFinder to group closely related LTRs. We processed the orthofinder data selecting orthogroups which were in equal representation on both subgenomes, and that were also present in Helianthus. After this, we aligned the selected orthogroups using mafft, and cleaned poorly aligned regions using Gblocks (Castresana 2000; Talavera and Castresana 2007), with not stringent options (i.e. "allow smaller final blocks", "allow gap positions within the final blocks", and "allow less strict flanking regions"). After this, we removed sequences with more than $50 \%$ missing data, and re-checked whether numbers of TEs were still balanced between subgenomes. We then re-aligned the data using mafft and inferred a tree for each ortholog. We kept only orthogroups where the $S$. atractyloides sequences were monophyletic, and where both subgenomes were nonmonophyletic. For the final set of orthogroups passing all this filtering, we calculated pairwise Jukes Cantor distance between each $S$. atractyloides LTR-region; and between each $S$. atractyloides and $H$. annuus. The Jukes Cantor distances were plotted in $\mathrm{R}$ and we analysed the overall frequency and converted it to million of years distance by a simple three rule with the Helianthus divergence with Scalesia of 6.14 Mya (Supplementary Information; Supplementary Figure 04).

\section{Signatures of selection and expanded gene regions}

Using the Scalesia genome together with the remaining Asteraceae genomes we ran CAFE analyses (De Bie et al. 2006; Mendes et al. 2020) to estimate significant gene family expansions and contractions. Briefly, we did an all-by-all BLAST to identify orthologues in the dataset and estimated significantly expanded and contracted families using CAFÉ. To interpret the data we relied on Gene Ontology Annotation. We obtained GOs for the annotated Scalesia genes by means of two complementary approaches: 1) by using the Interproscan command-line version (Jones et al. 2014), using the NCBI's Conserved Domains Database (CDD), Prediction of Coiled Coil Regions in Proteins (COILS), Protein Information Resource (PIRSF), PRINTS, PFAM, ProDom, ProSitePatterns and ProSiteProfiles, the Structure-Function Linkage Database (SFLD), Simple Modular Architecture Research Tool (SMART), SUPERFAMILY, and TIGRFAMs databases; 2) by extracting the curated Swiss Prot database from UniProt (Viridiplantae). We blasted the Scalesia genes to this database and kept hits with an e-value below 1e-10. We then extracted the GOs from each gene from the database and assigned these to Scalesia's correspondent orthologs (e-value below 1e-10). Genes belonging to significantly expanded gene 
648 families in the $S$. atractyloides genome were analysed using a GO enrichment analysis. To do so, 649 we used the TopGO package using the 'elim' algorithm which takes GO hierarchy into account 650 (Alexa, Rahnenfuhrer, and Others 2010; Alexa and Rahnenführer 2009), this were then plotted 651 with REVIGO (Supek etal. 2011).

652 To test which genes are under positive selection in $S$. atractyloides genome, we retrieved 653 the orthogroups from all Asteraceae, and aligned the cds from each orthogroup using prank 654 (Löytynoja 2014). Considering the divergence in the genomes, as well as evidence for fast 655 evolution in Asteraceae genomes (including this paper), we ran zorro (Wu, Chatterji, and Eisen 656 2012), to assess alignments. Zorro scores each alignment position with a score between 0-10, 657 and we selected only alignments with an average score position of 5 or greater. For each of 658 these, we inferred a tree using iqtree and ran HyPhy using its aBSREL selection test (Smith et al. 659 2015; Pond, Frost, and Muse 2005). To summarize these results we: 1) ran a GO enrichment (as 660 specified above) and plotted results using REVIGO; 2) identified the Arabidopsis ortholog to 661 each of the Scalesiagene under selection using BLAST, and analysed the Arabidopsis literature 662 for that particular gene (Supplementary Information; Supplementary Table 07); 3) we ran a 663 STRING analysis using the Arabidopsis ortholog (Szklarczyk et al. 2015), exploring the potential 664 protein-protein interactions among genes under selection. Interaction scores of edges were 665 calculated based on the parameters Experiments, Co-expression, Neighborhood, Gene fusion 666 and Co-occurrence. Edges with interaction score higher than 0.400 were kept in the network. 667 After excluding genes with no physical connection, the STRING network had 627 nodes with 668470 edges (PPI enrichment p-value $<0.001$ ). To simplify the densely connected network into 669 potential biologically functional clusters, we used the distance matrix obtained from the STRING 670 global scores as the input to perform a k-Means clustering analysis (number of clusters $=6$ ). 4 671 out of the 6 clusters are enriched for biological processes related GO terms. Cluster 1 (red 672 bubbles) were enriched for the GO term metabolic processes, cluster 3 (lime green bubbles) for 673 histone modifications and chromosome organization, cluster 4 (green bubbles) for response to 674 light, and cluster 6 (purple bubbles) for ribosomal large subunit biogenesis and RNA-

675 processing.

676

\section{Data Availability}

We are in the process of submitting raw reads to ENA and the reference genome to

680 Dryad.

\section{Author contributions}

J.Ce. designed the experiment, processed and analysed the data and wrote the manuscript. B.P., J.M.L.G., A. R.-C., J.Ca., S.B., J.V., S.L., D.M. helped analysed the data. J.L. was responsible for flow cytometry analyses. C.K., L. S.-B. helped retrieving DNA/RNA, N. W., M.N., P.J.D.,G.R.-T. obtained permits. M.F.-M., P.V., R.M. obtained the outgroups. G.P., A.S., N.S., N.R.N., O.S., M.T.P.G., J.H. L.-M., L.R. contributed with senior expertise in data generation and interpretation, Alexander Suh22,23. M.D.M. obtained funding, supervised J.C. and wrote the manuscript. All the authors revised and approved the manuscript.

\section{Acknowledgements}

JC is grateful to Simen R. Sandve for fruitful discussion, Martin LaForest for sharing genome annotations for his organism, and Henning Adsersen for botanical expertise and logistical support in utilizing the University of Copenhagen botanical collections. Jennifer Mandel kindly shared the Asteraceae COS. The collection and photography of specimens, and 
695

696

697

698

699

700

701

702

703

704

705

706

707

708

709

710

711

712

713

714

715

716

717

718

719

720

721

722

723

724

725

726

727

728

729

730

731

732

733

734

735

736

737

738

739

740

741

742

743

744

745 the preparation of this manuscript benefited enormously from the cooperative assistance of the personnel of the Charles Darwin Foundation Research Station, who made arrangements for collecting trips, arranged laboratory space, and offered encouragement and support throughout the project. Scalesia specimens were collected under the Galápagos National Park research permit number PC-001/98 PNG and MAAE-DBI-CM-2021-0213. This publication is contribution number 2426 of the Charles Darwin Foundation for the Galápagos Islands. This work was supported by the Norwegian Research Council via project number 287327 awarded to MDM.

\section{References}

Alexa, Adrian, and Jörg Rahnenführer. 2009. “Gene Set Enrichment Analysis with topGO.” Bioconductor Improv 27: 1-26.

Alexa, Adrian, Jorg Rahnenfuhrer, and Others. 2010. “topGO: Enrichment Analysis for Gene Ontology." R Package Version 2 (0): 2010.

Alger, Elizabeth I., and Patrick P. Edger. 2020. “One Subgenome to Rule Them All: Underlying Mechanisms of Subgenome Dominance." Current Opinion in Plant Biology 54 (April): 10813.

Allen, Micah, Davide Poggiali, Kirstie Whitaker, Tom Rhys Marshall, and Rogier A. Kievit. 2019. "Raincloud Plots: A Multi-Platform Tool for Robust Data Visualization." Wellcome Open Research 4 (April): 63.

Andrews, S. 2017. “FastQC: A Quality Control Tool for High Throughput Sequence Data. 2010."

An, Zengxuan, Liufan Yin, Yuhao Liu, Maolin Peng, Wen-Hui Shen, and Aiwu Dong. 2020. "The Histone Methylation Readers MRG1/MRG2 and the Histone Chaperones NRP1/NRP2 Associate in Fine-Tuning Arabidopsis Flowering Time." The Plant Journal: For Cell and Molecular Biology 103 (3): 1010-24.

Badouin, Hélène, Jérôme Gouzy, Christopher J. Grassa, Florent Murat, S. Evan Staton, Ludovic Cottret, Christine Lelandais-Brière, et al. 2017. “The Sunflower Genome Provides Insights into Oil Metabolism, Flowering and Asterid Evolution." Nature 546 (7656): 148-52.

Baeckens, Simon, and Raoul Van Damme. 2020. “The Island Syndrome." Current Biology: CB 30 (8): R338-39.

Baldwin, B. G., and M. J. Sanderson. 1998. "Age and Rate of Diversification of the Hawaiian Silversword Alliance (Compositae)." Proceedings of the National Academy of Sciences of the United States of America 95 (16): 9402-6.

Basset, Gilles J. C., Stéphane Ravanel, Eoin P. Quinlivan, Ruth White, James J. Giovannoni, Fabrice Rébeillé, Brian P. Nichols, et al. 2004. "Folate Synthesis in Plants: The Last Step of the PAminobenzoate Branch Is Catalyzed by a Plastidial Aminodeoxychorismate Lyase." The Plant Journal: For Cell and Molecular Biology 40 (4): 453-61.

Berckmans, Barbara, Tim Lammens, Hilde Van Den Daele, Zoltan Magyar, Laszlo Bögre, and Lieven De Veylder. 2011. "Light-Dependent Regulation of DEL1 Is Determined by the Antagonistic Action of E2Fb and E2Fc." Plant Physiology 157 (3): 1440-51.

Besse, Pascale, Denis DaSilva, Laurence Humeau, Joyce Govinden-Soulange, Ameenah GuribFakim, and Hippolyte Kodja. 2003. "A Genetic Diversity Study of Endangered Psiadia Species Endemic from Mauritius Island Using PCR Markers." Biochemical Systematics and Ecology 31 (12): 1427-45.

Bird, Kevin A., Chad E. Niederhuth, Shujun Ou, Malia Gehan, J. Chris Pires, Zhiyong Xiong, Robert VanBuren, and Patrick P. Edger. 2021. "Replaying the Evolutionary Tape to Investigate Subgenome Dominance in Allopolyploid Brassica Napus." The New Phytologist230 (1): 354-71.

Bird, Kevin A., Robert VanBuren, Joshua R. Puzey, and Patrick P. Edger. 2018. "The Causes and Consequences of Subgenome Dominance in Hybrids and Recent Polyploids." The New Phytologist 220 (1): 87-93.

Bittner, Andras, Bettina Hause, and Margarete Baier. 2021. “Cold-Priming Causes Oxylipin 
746
Dampening during the Early Cold and Light Response of Arabidopsis Thaliana." Journal of Experimental Botany, June. https://doi.org/10.1093/jxb/erab314.

Blaschke, Jeremy D., and Roger W. Sanders. 2009. "PRELIMINARY INSIGHTS INTO THE PHYLOGENY AND SPECIATION OF SCALESIA (ASTERACEAE), GALÁPAGOS ISLANDS.” Journal of the Botanical Research Institute of Texas 3 (1): 177-91.

Bolger, Anthony M., Marc Lohse, and Bjoern Usadel. 2014. "Trimmomatic: A Flexible Trimmer for Illumina Sequence Data." Bioinformatics 30 (15): 2114-20.

Bradnam, Keith R., Joseph N. Fass, Anton Alexandrov, Paul Baranay, Michael Bechner, Inanç Birol, Sébastien Boisvert, et al. 2013. "Assemblathon 2: Evaluating de Novo Methods of Genome Assembly in Three Vertebrate Species." GigaScience 2 (1): 10.

Burns, Kevin C. 2019. Evolution in Isolation: The Search for an Island Syndrome in Plants. Cambridge University Press.

Castells, Enric, Jean Molinier, Giovanna Benvenuto, Clara Bourbousse, Gerald Zabulon, Antoine Zalc, Stefano Cazzaniga, Pascal Genschik, Fredy Barneche, and Chris Bowler. 2011. "The Conserved Factor DE-ETIOLATED 1 Cooperates with CUL4-DDB1DDB2 to Maintain Genome Integrity upon UV Stress." The EMBO Journal 30 (6): 1162-72.

Castresana, J. 2000. "Selection of Conserved Blocks from Multiple Alignments for Their Use in Phylogenetic Analysis.” Molecular Biology and Evolution 17 (4): 540-52.

Catchen, J. M., J. S. Conery, and J. H. Postlethwait. 2009. "Automated Identification of Conserved Synteny after Whole-Genome Duplication." Genome Research. https://doi.org/10.1101/gr.090480.108.

Chen, Donghong, Anne Molitor, Chunlin Liu, and Wen-Hui Shen. 2010. "The Arabidopsis PRC1like Ring-Finger Proteins Are Necessary for Repression of Embryonic Traits during Vegetative Growth." Cell Research 20 (12): 1332-44.

Chen, Siyu, Yuli Ding, Hainan Tian, Shucai Wang, and Yuelin Zhang. 2021. "WRKY54 and WRKY70 Positively Regulate SARD1 and CBP60g Expression in Plant Immunity." Plant Signaling \& Behavior 16 (10): 1932142.

Conway, Jake R., Alexander Lex, and Nils Gehlenborg. 2017. "UpSetR: An R Package for the Visualization of Intersecting Sets and Their Properties." Bioinformatics 33 (18): 2938-40.

Crane, Renee A., Marielle Cardénas Valdez, Nelly Castaneda, Charidan L. Jackson, Ciairra J. Riley, Islam Mostafa, Wenwen Kong, Shweta Chhajed, Sixue Chen, and Judy A. Brusslan. 2019. "Negative Regulation of Age-Related Developmental Leaf Senescence by the IAOx Pathway, PEN1, and PEN3." Frontiers in Plant Science 10 (October): 1202.

Crawford, Daniel J., Timothy K. Lowrey, Gregory J. Anderson, Gabriel Bernardello, Arnoldo Santos-Guerra, and Tod F. Stuessy. 2009. "Genetic Diversity in Asteraceae Endemic to Oceanic Islands: Baker's Law and Polyploidy.” Syst Evol Biogeogr Compos 139: 151.

Czarnocka, Weronika, Yosef Fichman, Maciej Bernacki, Elżbieta Różańska, Izabela SańkoSawczenko, Ron Mittler, and Stanisław Karpiński. 2020. "FMO1 Is Involved in Excess Light Stress-Induced Signal Transduction and Cell Death Signaling." Cells 9 (10). https://doi.org/10.3390/cells9102163.

Dal Bosco, Cristina, Lina Lezhneva, Alexander Biehl, Dario Leister, Heinrich Strotmann, Gerd Wanner, and Jorg Meurer. 2004. "Inactivation of the Chloroplast ATP Synthase Gamma Subunit Results in High Non-Photochemical Fluorescence Quenching and Altered Nuclear Gene Expression in Arabidopsis Thaliana." The Journal of Biological Chemistry 279 (2): 1060-69.

Danecek, Petr, James K. Bonfield, Jennifer Liddle, John Marshall, Valeriu Ohan, Martin O. Pollard, Andrew Whitwham, et al. 2021. "Twelve Years of SAMtools and BCFtools." GigaScience 10 (2). https://doi.org/10.1093/gigascience/giab008.

Darwin, B. Y. Charles, Es Q., R. S. F., L. S. F., G. S. F., and Alfred Wallace. n.d. "I. On the Tendency of Species to Form Varieties; And on the Perpetuation of Varieties and Species by Natural Means of Selection." Accessed June 18, 2021. http://anthro198.pbworks.com/f/Darwin+\&+Wallace+(1858)++ On+the+Tendency+of+Species+to+form+Varieties.pdf. Darwin, Charles. 1859. “On the Origin of Species by Means of Natural Selection, Or, The 
800

801

802

803

804

805

806

807

808

809

810

811

812

813

814

815

816

817

818

819

820

821

822

823

824

825

826

827

828

829

830

831

832

833

834

835

836

837

838

839

840

841

842

843

844

845

846

847

848

849

850

851

852

853
Preservation of Favoured Races in the Struggle for Life /."

https://doi.org/10.5962/bhl.title.68064.

De Bie, Tijl, Nello Cristianini, Jeffery P. Demuth, and Matthew W. Hahn. 2006. “CAFE: A Computational Tool for the Study of Gene Family Evolution.” Bioinformatics 22 (10): 1269-71.

Delcher, A. L., S. Kasif, R. D. Fleischmann, J. Peterson, O. White, and S. L. Salzberg. 1999. “Alignment of Whole Genomes." Nucleic Acids Research 27 (11): 2369-76.

Diop, Seydina I., Oliver Subotic, Alejandro Giraldo-Fonseca, Manuel Waller, Alexander Kirbis, Anna Neubauer, Giacomo Potente, et al. 2020. "A Pseudomolecule-Scale Genome Assembly of the Liverwort Marchantia Polymorpha." The Plant Journal: For Cell and Molecular Biology 101 (6): 1378-96.

Dolezel, Jaroslav, Sergio Sgorbati, and Sergio Lucretti. 1992. “Comparison of Three DNA Fluorochromes for Flow Cytometric Estimation of Nuclear DNA Content in Plants." Physiologia Plantarum. https://doi.org/10.1034/j.1399-3054.1992.850410.x.

Dolezel, J., J. Bartos, H. Voglmayr, and J. Greilhuber. 2003. "Nuclear DNA Content and Genome Size of Trout and Human." Cytometry. Part A: The Journal of the International Society for Analytical Cytology.

Douglas, Gavin M., Gesseca Gos, Kim A. Steige, Adriana Salcedo, Karl Holm, Emily B. Josephs, Ramesh Arunkumar, et al. 2015. "Hybrid Origins and the Earliest Stages of Diploidization in the Highly Successful Recent Polyploid Capsella Bursa-Pastoris." Proceedings of the National Academy of Sciences of the United States of America 112 (9): 2806-11.

Eastwood, A., M. Gibby, and Q. C. B. Cronk. 2004. "Evolution of St Helena Arborescent Astereae (Asteraceae): Relationships of the Genera Commidendrum and Melanodendron." Botanical Journal of the Linnean Society. Linnean Society of London 144 (1): 69-83.

Eddy, Sean. 1992. “HMMER User's Guide." Department of Genetics, Washington University School of Medicine 2 (1): 13.

Edger, Patrick P., Michael R. McKain, Kevin A. Bird, and Robert VanBuren. 2018. "Subgenome Assignment in Allopolyploids: Challenges and Future Directions." Current Opinion in Plant Biology 42 (April): 76-80.

Eliasson, Uno. 1974. “Studies in Galapagos Plants. XIV. The Genus Scalesia Arn.” Opera Botanica 36: 1-117.

Eliasson, Uno, and Others. 1974. “Studies in Galápagos Plants. XIV. The Genus Scalesia Arn.” https://pascalfrancis.inist.fr/vibad/index.php?action=getRecordDetail\&idt=PASCAL7537000304.

Eliasson, U., and Eliasson U. 1974. "Studies in Galapagos Plants. Xiv. The Genus Scalesia Arn." https://pascal-

francis.inist.fr/vibad/index.php?action=getRecordDetail\&idt=PASCAL7537000304.

Ellinghaus, David, Stefan Kurtz, and Ute Willhoeft. 2008. "LTRharvest, an Efficient and Flexible Software for de Novo Detection of LTR Retrotransposons.” BMC Bioinformatics 9 (January): 18.

Emerson, Brent C. 2008. “Speciation on Islands: What Are We Learning?” Biological Journal of the Linnean Society. Linnean Society of London 95 (1): 47-52.

Emms, David M., and Steven Kelly. 2015. "OrthoFinder: Solving Fundamental Biases in Whole Genome Comparisons Dramatically Improves Orthogroup Inference Accuracy." Genome Biology 16 (August): 157.

Endo, Motomu, Daiki Kudo, Tomoko Koto, Hanako Shimizu, and Takashi Araki. 2014. "LightDependent Destabilization of PHL in Arabidopsis." Plant Signaling \& Behavior 9 (3): e28118.

Endo, Motomu, Yoshiyasu Tanigawa, Tadashi Murakami, Takashi Araki, and Akira Nagatani. 2013. "PHYTOCHROME-DEPENDENT LATE-FLOWERING Accelerates Flowering through Physical Interactions with Phytochrome B and CONSTANS." Proceedings of the National Academy of Sciences of the United States of America 110 (44): 18017-22.

Faircloth, Brant C. 2016. "PHYLUCE Is a Software Package for the Analysis of Conserved Genomic Loci." Bioinformatics 32 (5): 786-88. 
854

855

856

857

858

859

860

861

862

863

864

865

866

867

868

869

870

871

872

873

874

875

876

877

878

879

880

881

882

883

884

885

886

887

888

889

890

891

892

893

894

895

896

897

898

899

900

901

902

903

904

905

906

907
Faircloth, Brant C., John E. McCormack, Nicholas G. Crawford, Michael G. Harvey, Robb T. Brumfield, and Travis C. Glenn. 2012. "Ultraconserved Elements Anchor Thousands of Genetic Markers Spanning Multiple Evolutionary Timescales." Systematic Biology 61 (5): 717-26.

Fal, Kateryna, Mengying Liu, Assem Duisembekova, Yassin Refahi, Elizabeth S. Haswell, and Olivier Hamant. 2017. "Phyllotactic Regularity Requires the Paf1 Complex in Arabidopsis." Development https://doi.org/10.1242/dev.154369.

Fernández-Mazuecos, Mario, Pablo Vargas, Ross A. McCauley, David Monjas, Ana Otero, Jaime A. Chaves, Juan Ernesto Guevara Andino, and Gonzalo Rivas-Torres. 2020. "The Radiation of Darwin's Giant Daisies in the Galápagos Islands.” Current Biology: CB 30 (24): 4989-98.e7.

Flynn, Jullien M., Robert Hubley, Clément Goubert, Jeb Rosen, Andrew G. Clark, Cédric Feschotte, and Arian F. Smit. 2020. "RepeatModeler2 for Automated Genomic Discovery of Transposable Element Families." Proceedings of the National Academy of Sciences of the United States of America 117 (17): 9451-57.

Fox, Ana Romina, Florencia Scochera, Timothée Laloux, Karolina Filik, Hervé Degand, Pierre Morsomme, Karina Alleva, and François Chaumont. 2020. "Plasma Membrane Aquaporins Interact with the Endoplasmic Reticulum Resident VAP27 Proteins at ER-PM Contact Sites and Endocytic Structures." The New Phytologist228 (3): 973-88.

Freeling, Michael, Michael J. Scanlon, and John E. Fowler. 2015. "Fractionation and Subfunctionalization Following Genome Duplications: Mechanisms That Drive Gene Content and Their Consequences." Current Opinion in Genetics \& Development. https://doi.org/10.1016/j.gde.2015.11.002.

Fukunaga, Satoshi, Miho Sogame, Masaki Hata, Suthitar Singkaravanit-Ogawa, Mariola Piślewska-Bednarek, Mariko Onozawa-Komori, Takumi Nishiuchi, et al. 2017. “Dysfunction of Arabidopsis MACPF Domain Protein Activates Programmed Cell Death via Tryptophan Metabolism in MAMP-Triggered Immunity." The Plant Journal. https://doi.org/10.1111/tpj.13391.

Fu, Mengni, Changshun Yuan, Aihua Song, Jun Lu, Xiaojing Wang, and Shulan Sun. 2019. "AtWDS1 Negatively Regulates Age-Dependent and Dark-Induced Leaf Senescence in Arabidopsis." Plant Science: An International Journal of Experimental Plant Biology 285 (August): 44-54.

Galbraith, D. W., K. R. Harkins, J. M. Maddox, N. M. Ayres, D. P. Sharma, and E. Firoozabady. 1983. "Rapid Flow Cytometric Analysis of the Cell Cycle in Intact Plant Tissues." Science 220 (4601): 1049-51.

Glass, Magdalena, Sarah Barkwill, Faride Unda, and Shawn D. Mansfield. 2015. “Endo- $\beta-1,4-$ Glucanases Impact Plant Cell Wall Development by Influencing Cellulose Crystallization." Journal of Integrative Plant Biology. https://doi.org/10.1111/jipb.12353.

Gómez-Zambrano, Ángeles, Pedro Crevillén, José M. Franco-Zorrilla, Juan A. López, Jordi Moreno-Romero, Pawel Roszak, Juan Santos-González, et al. 2018. “Arabidopsis SWC4 Binds DNA and Recruits the SWR1 Complex to Modulate Histone H2A.Z Deposition at Key Regulatory Genes." Molecular Plant11 (6): 815-32.

Greilhuber, Johann, Jaroslav Dolezel, Martin A. Lysák, and Michael D. Bennett. 2005. “The Origin, Evolution and Proposed Stabilization of the Terms 'Genome Size' and 'C-Value' to Describe Nuclear DNA Contents." Annals of Botany 95 (1): 255-60.

Gruenstaeudl, Michael, Arnoldo Santos-Guerra, and Robert K. Jansen. 2013. "Phylogenetic Analyses ofTolpisAdans. (Asteraceae) Reveal Patterns of Adaptive Radiation, Multiple Colonization and Interspecific Hybridization." Cladistics: The International Journal of the Willi Hennig Society 29 (4): 416-34.

Guan, Dengfeng, Shane A. McCarthy, Jonathan Wood, Kerstin Howe, Yadong Wang, and Richard Durbin. 2020. "Identifying and Removing Haplotypic Duplication in Primary Genome Assemblies." Bioinformatics 36 (9): 2896-98.

Hao, Zhaodong, Dekang Lv, Ying Ge, Jisen Shi, Dolf Weijers, Guangchuang Yu, and Jinhui Chen. 2020. "RIdeogram: Drawing SVG Graphics to Visualize and Map Genome-Wide Data on the Idiograms.” PeerJ. Computer Science 6 (January): e251. 
908

909

910

911

912

913

914

915

916

917

918

919

920

921

922

923

924

925

926

927

928

929

930

931

932

933

934

935

936

937

938

939

940

941

942

943

944

945

946

947

948

949

950

951

952

953

954

955

956

957

958

959

960

961
He, Y. 2004. "PAF1-Complex-Mediated Histone Methylation of FLOWERING LOCUS C Chromatin Is Required for the Vernalization-Responsive, Winter-Annual Habit in Arabidopsis." Genes \& Development https://doi.org/10.1101/gad.1244504.

Holding, D. 2000. "The Chloroplast and Leaf Developmental Mutant, Pale Cress, Exhibits LightConditional Severity and Symptoms Characteristic of Its ABA Deficiency." Annals of Botany. https://doi.org/10.1006/anbo.2000.1263.

Holt, Carson, and Mark Yandell. 2011. "MAKER2: An Annotation Pipeline and Genome-Database Management Tool for Second-Generation Genome Projects." BMC Bioinformatics 12 (December): 491.

Hoson, T., K. Soga, K. Wakabayashi, T. Hashimoto, I. Karahara, S. Yano, F. Tanigaki, et al. 2014. "Growth Stimulation in Inflorescences of an Arabidopsis Tubulin Mutant under Microgravity Conditions in Space." Plant Biology 16 Suppl 1 (January): 91-96.

Hove, Colette A. ten, Zoltán Bochdanovits, Vera M. A. Jansweijer, Fenne G. Koning, Lidija Berke, Gabino F. Sanchez-Perez, Ben Scheres, and Renze Heidstra. 2011. "Probing the Roles of LRR RLK Genes in Arabidopsis Thaliana Roots Using a Custom T-DNA Insertion Set." Plant Molecular Biology 76 (1): 69-83.

Husbands, Aman Y., Anna H. Benkovics, Fabio T. S. Nogueira, Mukesh Lodha, and Marja C. P. Timmermans. 2016. "The ASYMMETRIC LEAVES Complex Employs Multiple Modes of Regulation to Affect Adaxial-Abaxial Patterning and Leaf Complexity." The Plant Cell. https://doi.org/10.1105/tpc.15.00454.

Itow, Syuzo. 1995. "Phytogeography and Ecology of Scalesia (compositae) Endemic to the Galapagos Islands!" Pacific Science 49 (1): 17-30.

Jakoby, Marc J., Doris Falkenhan, Michael T. Mader, Ginger Brininstool, Elisabeth Wischnitzki, Nicole Platz, Andrew Hudson, Martin Hülskamp, John Larkin, and Arp Schnittger. 2008. “Transcriptional Profiling of Mature Arabidopsis Trichomes Reveals That NOECK Encodes the MIXTA-like Transcriptional Regulator MYB106." Plant Physiology 148 (3): 1583-1602.

Ji, Hao, Yanhui Peng, Nicole Meckes, Sara Allen, C. Neal Stewart Jr, and M. Brian Traw. 2014. “ATP-Dependent Binding Cassette Transporter G Family Member 16 Increases Plant Tolerance to Abscisic Acid and Assists in Basal Resistance against Pseudomonas Syringae DC3000." Plant Physiology 166 (2): 879-88.

Jones, Philip, David Binns, Hsin-Yu Chang, Matthew Fraser, Weizhong Li, Craig McAnulla, Hamish McWilliam, et al. 2014. "InterProScan 5: Genome-Scale Protein Function Classification." Bioinformatics 30 (9): 1236-40.

Kapos, Paul, Fang Xu, Thierry Meinnel, Carmela Giglione, and Xin Li. 2015. “N-Terminal Modifications Contribute to Flowering Time and Immune Response Regulations." Plant Signaling \& Behavior 10 (10): e1073874.

Keller, Oliver, Martin Kollmar, Mario Stanke, and Stephan Waack. 2011. "A Novel Hybrid Gene Prediction Method Employing Protein Multiple Sequence Alignments." Bioinformatics 27 (6): 757-63.

Kessler, Sharon A., Hiroko Shimosato-Asano, Nana F. Keinath, Samuel E. Wuest, Gwyneth Ingram, Ralph Panstruga, and Ueli Grossniklaus. 2010. “Conserved Molecular Components for Pollen Tube Reception and Fungal Invasion.” Science 330 (6006): 968-71.

Kim, Areum, Jilin Chen, Deepa Khare, Jun-Young Jin, Yasuyo Yamaoka, Masayoshi Maeshima, Yunde Zhao, Enrico Martinoia, Jae-Ung Hwang, and Youngsook Lee. 2020. “Non-Intrinsic ATP-Binding Cassette Proteins ABCI19, ABCI2 0 and ABCI21 Modulate Cytokinin Response at the Endoplasmic Reticulum in Arabidopsis Thaliana." Plant Cell Reports 39 (4): 473-87.

Kim, Joo Y., Su J. Park, Boseung Jang, Che-Hun Jung, Sung J. Ahn, Chang-Hyo Goh, Kyoungwon Cho, Oksoo Han, and Hunseung Kang. 2007. "Functional Characterization of a Glycine-Rich RNA-Binding Protein 2 in Arabidopsis Thaliana under Abiotic Stress Conditions." The Plant Journal. https://doi.org/10.1111/j.1365-313x.2007.03057.x.

Kim, S. C., D. J. Crawford, J. Francisco-Ortega, and A. Santos-Guerra. 1996. “A Common Origin for Woody Sonchus and Five Related Genera in the Macaronesian Islands: Molecular Evidence for Extensive Radiation." Proceedings of the National Academy of Sciences of the United States of America 93 (15): 7743-48. 
962

963

964

965

966

967

968

969

970

971

972

973

974

975

976

977

978

979

980

981

982

983

984

985

986

987

988

989

990

991

992

993

994

995

996

997

998

999

1000

1001

1002

1003

1004

1005

1006

1007

1008

1009

1010

1011

1012

1013

1014

1015
Kleine, Tatjana, Peter Kindgren, Catherine Benedict, Luke Hendrickson, and Asa Strand. 2007. "Genome-Wide Gene Expression Analysis Reveals a Critical Role for CRYPTOCHROME1 in the Response of Arabidopsis to High Irradiance." Plant Physiology 144 (3): 1391-1406.

Knope, Matthew L., M. Renee Bellinger, Erin M. Datlof, Timothy J. Gallaher, and Melissa A. Johnson. 2020. "Insights into the Evolutionary History of the Hawaiian Bidens (Asteraceae) Adaptive Radiation Revealed Through Phylogenomics." The Journal of Heredity111 (1): 119-37.

Knope, Matthew L., Clifford W. Morden, Vicki A. Funk, and Tadashi Fukami. 2012. “Area and the Rapid Radiation of Hawaiian Bidens (Asteraceae)." Journal of Biogeography39 (7): 120616.

Kojima, Shoko, Mayumi Iwasaki, Hiro Takahashi, Tomoya Imai, Yoko Matsumura, Delphine Fleury, Mieke Van Lijsebettens, Yasunori Machida, and Chiyoko Machida. 2011.

"Asymmetric leaves2 and Elongator, a Histone Acetyltransferase Complex, Mediate the Establishment of Polarity in Leaves of Arabidopsis Thaliana." Plant \& Cell Physiology 52 (8): 1259-73.

Korf, lan. 2004. “Gene Finding in Novel Genomes.” BMC Bioinformatics 5 (May): 59.

Krzywinski, Martin, Jacqueline Schein, Inanç Birol, Joseph Connors, Randy Gascoyne, Doug Horsman, Steven J. Jones, and Marco A. Marra. 2009. "Circos: An Information Aesthetic for Comparative Genomics." Genome Research 19 (9): 1639-45.

Kuki, Yasunobu, Ryoko Ohno, Kentaro Yoshida, and Shigeo Takumi. 2020. "Heterologous Expression of Wheat WRKY Transcription Factor Genes Transcriptionally Activated in Hybrid Necrosis Strains Alters Abiotic and Biotic Stress Tolerance in Transgenic Arabidopsis." Plant Physiology and Biochemistry: PPB / Societe Francaise de Physiologie Vegetale 150 (May): 71-79.

Kurtz, Stefan, Adam Phillippy, Arthur L. Delcher, Michael Smoot, Martin Shumway, Corina Antonescu, and Steven L. Salzberg. 2004. "Versatile and Open Software for Comparing Large Genomes.” Genome Biology 5 (2): R12.

Laetsch, Dominik R., and Mark L. Blaxter. 2017a. "BlobTools: Interrogation of Genome Assemblies." F1000Research 6 (1287): 1287.

- _ 2017b. "KinFin: Software for Taxon-Aware Analysis of Clustered Protein Sequences." G3 7 (10): 3349-57.

Laforest, Martin, Sara L. Martin, Katherine Bisaillon, Brahim Soufiane, Sydney Meloche, and Eric Page. 2020. "A Chromosome-Scale Draft Sequence of the Canada Fleabane Genome." Pest Management Science 76 (6): 2158-69.

Lahari, Triparna, Janelle Lazaro, Jeffrey M. Marcus, and Dana F. Schroeder. 2018. “RAD7 Homologues Contribute to Arabidopsis UV Tolerance." Plant Science: An International Journal of Experimental Plant Biology 277 (December): 267-77.

Lang, Daniel, Kristian K. Ullrich, Florent Murat, Jörg Fuchs, Jerry Jenkins, Fabian B. Haas, Mathieu Piednoel, et al. 2018. “ThePhyscomitrella Patenschromosome-Scale Assembly Reveals Moss Genome Structure and Evolution." The Plant Journal: For Cell and Molecular Biology 93 (3): 515-33.

Lawesson, Jonas Erik. 1988. "Stand-Level Dieback and Regeneration of Forests in the Galápagos Islands." Temporal and Spatial Patterns of Vegetation Dynamics. https://doi.org/10.1007/978-94-009-2275-4_10.

Li, Fay-Wei, Tomoaki Nishiyama, Manuel Waller, Eftychios Frangedakis, Jean Keller, Zheng Li, Noe Fernandez-Pozo, et al. 2020. "Anthoceros Genomes Illuminate the Origin of Land Plants and the Unique Biology of Hornworts." Nature Plants 6 (3): 259-72.

Li, Gang, Hamad Siddiqui, Yibo Teng, Rongcheng Lin, Xiang-Yuan Wan, Jigang Li, On-Sun Lau, et al. 2011. "Coordinated Transcriptional Regulation Underlying the Circadian Clock in Arabidopsis." Nature Cell Biology 13 (5): 616-22.

Li, H., and R. Durbin. 2009. "Fast and Accurate Short Read Alignment with Burrows-Wheeler Transform.” Bioinformatics. https://doi.org/10.1093/bioinformatics/btp324.

Li, Heng, and Richard Durbin. 2011. "Inference of Human Population History from Individual Whole-Genome Sequences.” Nature 475 (7357): 493-96. 
1016

1017

1018

1019

1020

1021

1022

1023

1024

1025

1026

1027

1028

1029

1030

1031

1032

1033

1034

1035

1036

1037

1038

1039

1040

1041

1042

1043

1044

1045

1046

1047

1048

1049

1050

1051

1052

1053

1054

1055

1056

1057

1058

1059

1060

1061

1062

1063

1064

1065

1066

1067

1068

1069
Li, Jian, Zheng Wang, Yugang Hu, Ying Cao, and Ligeng Ma. 2017. “Polycomb Group Proteins RING1A and RING1B Regulate the Vegetative Phase Transition in Arabidopsis." Frontiers in Plant Science 8 (May): 867.

Liu, Bo, Jian Yan, Weihua Li, Lijuan Yin, Ping Li, Hanxia Yu, Longsheng Xing, et al. 2020. "Mikania Micrantha Genome Provides Insights into the Molecular Mechanism of Rapid Growth." Nature Communications 11 (1): 340.

Liu, Huanhuan, Bao Liu, Shangling Lou, Hao Bi, Hu Tang, Shaofei Tong, Yan Song, et al. 2021. "CHYR1 Ubiquitinates the Phosphorylated WRKY70 for Degradation to Balance Immunity in Arabidopsis Thaliana." New Phytologist https://doi.org/10.1111/nph.17231.

Llorens, Carlos, Ricardo Futami, Laura Covelli, Laura Domínguez-Escribá, Jose M. Viu, Daniel Tamarit, Jose Aguilar-Rodríguez, et al. 2011. “The Gypsy Database (GyDB) of Mobile Genetic Elements: Release 2.0." Nucleic Acids Research 39 (Database issue): D70-74.

Lomolino, Mark V., Brett R. Riddle, and Robert J. Whittaker. 2017. “Biogeography.” https://digitalscholarship.unlv.edu/sls_fac_articles/433/.

Loureiro, João, Eleazar Rodriguez, Jaroslav Dolezel, and Conceição Santos. 2007. "Two New Nuclear Isolation Buffers for Plant DNA Flow Cytometry: A Test with 37 Species." Annals of Botany 100 (4): 875-88.

Lovell, John T., Alice H. MacQueen, Sujan Mamidi, Jason Bonnette, Jerry Jenkins, Joseph D. Napier, Avinash Sreedasyam, et al. 2021. "Genomic Mechanisms of Climate Adaptation in Polyploid Bioenergy Switchgrass.” Nature 590 (7846): 438-44.

Löytynoja, Ari. 2014. "Phylogeny-Aware Alignment with PRANK." Methods in Molecular Biology 1079: 155-70.

Mandel, Jennifer R., Rebecca B. Dikow, Vicki A. Funk, Rishi R. Masalia, S. Evan Staton, Alex Kozik, Richard W. Michelmore, Loren H. Rieseberg, and John M. Burke. 2014. “A Target Enrichment Method for Gathering Phylogenetic Information from Hundreds of Loci: An Example from the Compositae." Applications in Plant Sciences 2 (2). https://doi.org/10.3732/apps.1300085.

Mandel, Jennifer R., Rebecca B. Dikow, Carolina M. Siniscalchi, Ramhari Thapa, Linda E. Watson, and Vicki A. Funk. 2019. "A Fully Resolved Backbone Phylogeny Reveals Numerous Dispersals and Explosive Diversifications throughout the History of Asteraceae." Proceedings of the National Academy of Sciences of the United States of America 116 (28): 14083-88.

Marçais, Guillaume, and Carl Kingsford. 2011. "A Fast, Lock-Free Approach for Efficient Parallel Counting of Occurrences of K-Mers." Bioinformatics 27 (6): 764-70.

Markakis, Marios Nektarios, Tinne De Cnodder, Michal Lewandowski, Damien Simon, Agnieszka Boron, Daria Balcerowicz, Thanaa Doubbo, et al. 2012. "Identification of Genes Involved in the ACC-Mediated Control of Root Cell Elongation in Arabidopsis Thaliana." BMC Plant Biology. https://doi.org/10.1186/1471-2229-12-208.

Mayr, E. 1942. "Systematics and the Origin of Species from the Viewpoint of a Zoologist, Columbia Uni." Press, New York.

Ma, Zhaoxue, Wenjuan Wu, Weihua Huang, and Jirong Huang. 2015. “Down-Regulation of Specific Plastid Ribosomal Proteins Suppresses thf1 Leaf Variegation, Implying a Role of THF1 in Plastid Gene Expression." Photosynthesis Research 126 (2-3): 301-10.

Mendes, Fábio K., Dan Vanderpool, Ben Fulton, and Matthew W. Hahn. 2020. "CAFE 5 Models Variation in Evolutionary Rates among Gene Families." Bioinformatics , December. https://doi.org/10.1093/bioinformatics/btaa1022.

Meudt, Heidi M., Dirk C. Albach, Andrew J. Tanentzap, Javier Igea, Sophie C. Newmarch, Angela J. Brandt, William G. Lee, and Jennifer A. Tate. 2021. "Polyploidy on Islands: Its Emergence and Importance for Diversification." Frontiers in Plant Science 12 (March): 637214.

Meurer, J., C. Grevelding, P. Westhoff, and B. Reiss. 1998. "The PAC Protein Affects the Maturation of Specific Chloroplast mRNAs in Arabidopsis Thaliana." Molecular and General Genetics $M G G$. https://doi.org/10.1007/s004380050740.

Meurer, Jörg, Lisa-Marie Schmid, Rhea Stoppel, Dario Leister, Andreas Brachmann, and Nikolay Manavski. 2017. "PALE CRESS Binds to Plastid RNAs and Facilitates the Biogenesis of the 
1070

1071

1072

1073

1074

1075

1076

1077

1078

1079

1080

1081

1082

1083

1084

1085

1086

1087

1088

1089

1090

1091

1092

1093

1094

1095

1096

1097

1098

1099

1100

1101

1102

1103

1104

1105

1106

1107

1108

1109

1110

1111

1112

1113

1114

1115

1116

1117

1118

1119

1120

1121

1122

1123
50S Ribosomal Subunit." The Plant Journal. https://doi.org/10.1111/tpj.13662.

Mitros, Therese, Adam M. Session, Brandon T. James, Guohong Albert Wu, Mohammad B. Belaffif, Lindsay V. Clark, Shengqiang Shu, et al. 2020. "Genome Biology of the Paleotetraploid Perennial Biomass Crop Miscanthus." Nature Communications 11 (1): 5442.

Mitsui, Yuki, and Hiroaki Setoguchi. 2012. "Recent Origin and Adaptive Diversification of Ainsliaea (Asteraceae) in the Ryukyu Islands: Molecular Phylogenetic Inference Using Nuclear Microsatellite Markers." Osterreichische Botanische Zeitschrift298 (5): 985-96.

Moore, B., C. Holt, A. S. Alvarado, and M. Yandell. 2008. "MAKER: An Easy-to-Use Annotation Pipeline Designed for Emerging Model Organism Genomes." Genome / National Research Council Canada = Genome / Conseil National de Recherches Canada. https://genome.cshlp.org/content/18/1/188.short.

Noh, Seong Woo, Ri-Ra Seo, Hee Jin Park, and Ho Won Jung. 2021. “Two Arabidopsis Homologs of Human Lysine-Specific Demethylase Function in Epigenetic Regulation of Plant Defense Responses." Frontiers in Plant Science 12 (June): 688003.

Noutoshi, Yoshiteru, Takashi Kuromori, Takuji Wada, Takashi Hirayama, Asako Kamiya, Yuko Imura, Michiko Yasuda, Hideo Nakashita, Ken Shirasu, and Kazuo Shinozaki. 2006. "Loss of Necrotic Spotted Lesions 1 Associates with Cell Death and Defense Responses in Arabidopsis Thaliana." Plant Molecular Biology 62 (1-2): 29-42.

Ono, Mikio. 1967. "Chromosome Number of Scalesia (Compositae), an Endemic Genus of the Galapagos Islands." Journal of Japanese Botany 42 (12): 353-60.

Oravecz, Attila, Alexander Baumann, Zoltán Máté, Agnieszka Brzezinska, Jean Molinier, Edward J. Oakeley, Eva Adám, Eberhard Schäfer, Ferenc Nagy, and Roman Ulm. 2006. "CONSTITUTIVELY PHOTOMORPHOGENIC1 Is Required for the UV-B Response in Arabidopsis." The Plant Cell 18 (8): 1975-90.

Őszi, Erika, Csaba Papdi, Binish Mohammed, Aladár Petkó-Szandtner, Tünde Leviczky, Eszter Molnár, Carlos Galvan-Ampudia, et al. 2020. “E2FB Interacts with RETINOBLASTOMA RELATED and Regulates Cell Proliferation during Leaf Development." Plant Physiology 182 (1): 518-33.

Parra, Genis, Keith Bradnam, and Ian Korf. 2007. “CEGMA: A Pipeline to Accurately Annotate Core Genes in Eukaryotic Genomes." Bioinformatics 23 (9): 1061-67.

Peona, Valentina, Matthias H. Weissensteiner, and Alexander Suh. 2018. "How Complete Are 'complete' Genome Assemblies?-An Avian Perspective." Molecular Ecology Resources 18 (6): 1188-95.

Pond, Sergei L. Kosakovsky, Simon D. W. Frost, and Spencer V. Muse. 2005. “HyPhy: Hypothesis Testing Using Phylogenies." Bioinformatics 21 (5): 676-79.

Putnam, Nicholas H., Brendan L. O'Connell, Jonathan C. Stites, Brandon J. Rice, Marco Blanchette, Robert Calef, Christopher J. Troll, et al. 2016. "Chromosome-Scale Shotgun Assembly Using an in Vitro Method for Long-Range Linkage." Genome Research 26 (3): 342-50.

Renny-Byfield, Simon, Lei Gong, Joseph P. Gallagher, and Jonathan F. Wendel. 2015. "Persistence of Subgenomes in Paleopolyploid Cotton after 60 My of Evolution." Molecular Biology and Evolution 32 (4): 1063-71.

Reyes-Chin-Wo, Sebastian, Zhiwen Wang, Xinhua Yang, Alexander Kozik, Siwaret Arikit, Chi Song, Liangfeng Xia, et al. 2017. "Genome Assembly with in Vitro Proximity Ligation Data and Whole-Genome Triplication in Lettuce." Nature Communications 8 (April): 14953.

Ruan, Jue, and Heng Li. 2020. "Fast and Accurate Long-Read Assembly with wtdbg2." Nature Methods 17 (2): 155-58.

Sanderson, Michael J. 2003. "r8s: Inferring Absolute Rates of Molecular Evolution and Divergence Times in the Absence of a Molecular Clock." Bioinformatics 19 (2): 301-2.

Sasaki-Sekimoto, Yuko, Yusuke Jikumaru, Takeshi Obayashi, Hikaru Saito, Shinji Masuda, Yuji Kamiya, Hiroyuki Ohta, and Ken Shirasu. 2013. “Basic Helix-Loop-Helix Transcription Factors JASMONATE-ASSOCIATED MYC2-LIKE1 (JAM1), JAM2, and JAM3 Are Negative Regulators of Jasmonate Responses in Arabidopsis." Plant Physiology.

https://doi.org/10.1104/pp.113.220129. 
1124

1125

1126

1127

1128

1129

1130

1131

1132

1133

1134

1135

1136

1137

1138

1139

1140

1141

1142

1143

1144

1145

1146

1147

1148

1149

1150

1151

1152

1153

1154

1155

1156

1157

1158

1159

1160

1161

1162

1163

1164

1165

1166

1167

1168

1169

1170

1171

1172

1173

1174

1175

1176

1177
Schilling, Edward E., José L. Panero, and Uno H. Eliasson. 1994. “Evidence from Chloroplast DNA Restriction Site Analysis on the Relationships of Scalesia (Asteraceae: Heliantheae)." American Journal of Botany 81 (2): 248-54.

Schubert, Mikkel, Stinus Lindgreen, and Ludovic Orlando. 2016. “AdapterRemoval v2: Rapid Adapter Trimming, Identification, and Read Merging." BMC Research Notes 9 (February): 88.

Seppey, Mathieu, Mosè Manni, and Evgeny M. Zdobnov. 2019. "BUSCO: Assessing Genome Assembly and Annotation Completeness." Methods in Molecular Biology 1962: 227-45.

Session, Adam M., Yoshinobu Uno, Taejoon Kwon, Jarrod A. Chapman, Atsushi Toyoda, Shuji Takahashi, Akimasa Fukui, et al. 2016. "Genome Evolution in the Allotetraploid Frog Xenopus Laevis.” Nature 538 (7625): 336-43.

Shen, Lisha, Zhonghui Thong, Ximing Gong, Qing Shen, Yinbo Gan, and Hao Yu. 2014. “The Putative PRC1 RING-Finger Protein AtRING1A Regulates Flowering through Repressing MADS AFFECTING FLOWERING Genes in Arabidopsis." Development 141 (6): 1303-12.

Simão, Felipe A., Robert M. Waterhouse, Panagiotis Ioannidis, Evgenia V. Kriventseva, and Evgeny M. Zdobnov. 2015. "BUSCO: Assessing Genome Assembly and Annotation Completeness with Single-Copy Orthologs." Bioinformatics 31 (19): 3210-12.

Singh, Surjit, Sakthivel Kailasam, Jing-chi Lo, and Kuo-chen Yeh. 2021. "Histone H3 lysine4 Trimethylation-regulated GRF11 Expression Is Essential for the Iron-deficiency Response in Arabidopsis Thaliana." New Phytologist https://doi.org/10.1111/nph.17130.

Smeekens, Sjef. 2006. "Faculty Opinions Recommendation of Large-Scale Analysis of mRNA Translation States during Sucrose Starvation in Arabidopsis Cells Identifies Cell Proliferation and Chromatin Structure as Targets of Translational Control." Faculty Opinions - Post-Publication Peer Review of the Biomedical Literature. https://doi.org/10.3410/f.1032260.373846.

Smit, A., R. Hubley, and P. Green. 2013. “RepeatMasker 4.0.” Seattle, WA: Institute for Systems Biology.

Smith, Martin D., Joel O. Wertheim, Steven Weaver, Ben Murrell, Konrad Scheffler, and Sergei L. Kosakovsky Pond. 2015. "Less Is More: An Adaptive Branch-Site Random Effects Model for Efficient Detection of Episodic Diversifying Selection." Molecular Biology and Evolution 32 (5): 1342-53.

Spring, Otmar, Norbert Heil, and Bernhard Vogler. 1997. "Sesquiterpene Lactones and Flavanones in Scalesia Species." Phytochemistry 46 (8): 1369-73.

Steinbiss, Sascha, Ute Willhoeft, Gordon Gremme, and Stefan Kurtz. 2009. "Fine-Grained Annotation and Classification of de Novo Predicted LTR Retrotransposons." Nucleic Acids Research 37 (21): 7002-13.

Stöcklin, Jürg. 2009. “Darwin and the Plants of the Galápagos-Islands.” Bauhinia, 33-48.

Suda, Jan, Anna Krahulcová, Pavel Trávnícek, Radka Rosenbaumová, Tomás Peckert, and Frantisek Krahulec. 2007. "Genome Size Variation and Species Relationships in Hieracium Sub-Genus Pilosella (Asteraceae) as Inferred by Flow Cytometry." Annals of Botany 100 (6): 1323-35.

Suda, J., T. Kyncl, and V. Jarolímová. 2005. “Genome Size Variation in Macaronesian Angiosperms: Forty Percent of the Canarian Endemic Flora Completed." Plant Systematics and Evolution = Entwicklungsgeschichte Und Systematik Der Pflanzen 252 (3): 215-38.

Supek, Fran, Matko Bošnjak, Nives Škunca, and Tomislav Šmuc. 2011. "REVIGO Summarizes and Visualizes Long Lists of Gene Ontology Terms.” PIoS One 6 (7): e21800.

Swain, Swadhin, Nidhi Singh, and Ashis Kumar Nandi. 2015. "Identification of Plant Defence Regulators through Transcriptional Profiling of Arabidopsis Thaliana cdd1 Mutant." Journal of Biosciences 40 (1): 137-46.

Szklarczyk, Damian, Andrea Franceschini, Stefan Wyder, Kristoffer Forslund, Davide Heller, Jaime Huerta-Cepas, Milan Simonovic, et al. 2015. "STRING v10: Protein-Protein Interaction Networks, Integrated over the Tree of Life." Nucleic Acids Research 43 (Database issue): D447-52.

Talavera, Gerard, and Jose Castresana. 2007. “Improvement of Phylogenies after Removing 
1178

1179

1180

1181

1182

1183

1184

1185

1186

1187

1188

1189

1190

1191

1192

1193

1194

1195

1196

1197

1198

1199

1200

1201

1202

1203

1204

1205

1206

1207

1208

1209

1210

1211

1212

1213

1214

1215

1216

1217

1218

1219

1220

1221

1222

1223

1224

1225

1226

1227

1228

1229
Divergent and Ambiguously Aligned Blocks from Protein Sequence Alignments." Systematic Biology 56 (4): 564-77.

Tan, Yew-Foon, Nicholas O’Toole, Nicolas L. Taylor, and A. Harvey Millar. 2010. “Divalent Metal Ions in Plant Mitochondria and Their Role in Interactions with Proteins and Oxidative Stress-Induced Damage to Respiratory Function." Plant Physiology 152 (2): 747-61.

Tardaguila, Manuel, Lorena de la Fuente, Cristina Marti, Cécile Pereira, Francisco Jose PardoPalacios, Hector Del Risco, Marc Ferrell, et al. 2018. "Corrigendum: SQANTI: Extensive Characterization of Long-Read Transcript Sequences for Quality Control in Full-Length Transcriptome Identification and Quantification." Genome Research 28 (7): 1096.

Valverde, Federico, Andrew Groover, and José M. Romero. 2018. Evolution of Gene Regulatory Networks in Plant Development. Frontiers Media SA.

Vitales, Daniel, Teresa Garnatje, Jaume Pellicer, Joan Vallès, Arnoldo Santos-Guerra, and Isabel Sanmartín. 2014. "The Explosive Radiation of Cheirolophus (Asteraceae, Cardueae) in Macaronesia." BMC Evolutionary Biology 14 (June): 118.

Wallace, Alfred Russel. 1962. The Malay Archipelago: The Land of the Orang-Utan and the Bird of Paradise; a Narrative of Travel, with Studies of Man and Nature. Courier Corporation.

Wang, Pengwei, Roman Pleskot, Jingze Zang, Joanna Winkler, Jie Wang, Klaas Yperman, Tong Zhang, et al. 2019. "Plant AtEH/Pan1 Proteins Drive Autophagosome Formation at ER-PM Contact Sites with Actin and Endocytic Machinery." Nature Communications 10 (1): 5132.

Wang, Zhen, Fuxing Wang, Yechun Hong, Jirong Huang, Huazhong Shi, and Jian-Kang Zhu. 2016. 'Two Chloroplast Proteins Suppress Drought Resistance by Affecting ROS Production in Guard Cells." Plant Physiology 172 (4): 2491-2503.

Waterhouse, Robert M., Mathieu Seppey, Felipe A. Simão, Mosè Manni, Panagiotis Ioannidis, Guennadi Klioutchnikov, Evgenia V. Kriventseva, and Evgeny M. Zdobnov. 2018. "BUSCO Applications from Quality Assessments to Gene Prediction and Phylogenomics." Molecular Biology and Evolution 35 (3): 543-48.

White, Oliver W., Alfredo Reyes-Betancort, Mark A. Chapman, and Mark A. Carine. 2018. "Independent Homoploid Hybrid Speciation Events in the Macaronesian Endemic Genus Argyranthemum." Molecular Ecology 27 (23): 4856-74.

White, Oliver W., J. Alfredo Reyes-Betancort, Mark A. Chapman, and Mark A. Carine. 2020. "Geographical Isolation, Habitat Shifts and Hybridisation in the Diversification of the Macaronesian Endemic Genus Argyranthemum (Asteraceae)." The New Phytologist 228 (6): 1953-71.

Whitewoods, Chris D., Joseph Cammarata, Zoe Nemec Venza, Stephanie Sang, Ashley D. Crook, Tsuyoshi Aoyama, Xiao Y. Wang, et al. 2020. "CLAVATA Was a Genetic Novelty for the Morphological Innovation of 3D Growth in Land Plants." Current Biology: CB 30 (13): 2645-48.

Whitkus, Richard. 1998. "Genetics of Adaptive Radiation in Hawaiian and Cook Islands Species of Tetramolopium (Asteraceae). II. Genetic Linkage Map and Its Implications for Interspecific Breeding Barriers." Genetics. https://doi.org/10.1093/genetics/150.3.1209.

Whittaker, Robert J., School of Geography Robert J Whittaker, and José Maria FernandezPalacios. 2007. Island Biogeography: Ecology, Evolution, and Conservation. OUP Oxford.

Wolfe, K. H. 2001. "Yesterday's Polyploids and the Mystery of Diploidization.” Nature Reviews. Genetics 2 (5): 333-41.

Wu, Martin, Sourav Chatterji, and Jonathan A. Eisen. 2012. "Accounting for Alignment Uncertainty in Phylogenomics." PloS One 7 (1): e30288.

Xiong, Xue, Deyang Xu, Zhongnan Yang, Hai Huang, and Xiaofeng Cui. 2013. "A Single AminoAcid Substitution at Lysine 40 of an Arabidopsis Thaliana $\alpha$-Tubulin Causes Extensive Cell Proliferation and Expansion Defects." Journal of Integrative Plant Biology 55 (3): 209-20.

Zhang, Bangyue, Jianheng Jia, Min Yang, Chunxia Yan, and Yuzhen Han. 2012. "Overexpression of a LAM Domain Containing RNA-Binding Protein LARP1c Induces Precocious Leaf Senescence in Arabidopsis." Molecules and Cells 34 (4): 367-74. 